\title{
Dynamic Analysis of a Fractional-Order Model for Hepatitis B Virus with Holling II Functional Response
}

\author{
Ruiqing Shi $\mathbb{D}$, Ting Lu, and Cuihong Wang $\mathbb{D}$ \\ School of Mathematics and Computer Science, Shanxi Normal University, Linfen 041004, China \\ Correspondence should be addressed to Ruiqing Shi; shirq1979@163.com
}

Received 2 April 2019; Revised 15 July 2019; Accepted 24 July 2019; Published 6 August 2019

Academic Editor: Átila Bueno

Copyright (C) 2019 Ruiqing Shi et al. This is an open access article distributed under the Creative Commons Attribution License, which permits unrestricted use, distribution, and reproduction in any medium, provided the original work is properly cited.

\begin{abstract}
In this paper, a fractional-order model is constructed to describe the transmission of Hepatitis B Virus (HBV). Firstly, the existence and uniqueness of positive solutions are proved. Secondly, the basic reproduction number and the sufficient conditions for the existence of two equilibriums are obtained. Thirdly, the stability of equilibriums are analyzed. After that, some numerical simulations are performed to verify the theoretical prediction. Finally, a brief discussion is presented.
\end{abstract}

\section{Introduction}

In recent years, more and more attention has been paid to the research of epidemic disease, and it is a great challenge to control the spread of epidemic disease among people. Mathematical models of infectious disease are important tools, which provided theoretical basis for the prevention and control of disease[1-6]. Therefore, many scholars have established mathematical models to simulate the epidemic dynamics of virus in host cells. Nowak and Bangham presented a basic model of the dynamics of HIV infection[7, 8]. This model have since been used for other viral infections, like HBV [9-11] and HCV [12, 13]. The basic model can be formulated as a system of three differential equations:

$$
\begin{aligned}
& \frac{\mathrm{d} x}{\mathrm{~d} t}=\Pi-\delta x-\beta x v, \\
& \frac{\mathrm{d} y}{\mathrm{~d} t}=\beta x v-b y, \\
& \frac{\mathrm{d} v}{\mathrm{~d} t}=c y-\gamma v,
\end{aligned}
$$

where $x, y$, and $v$ are the densities of uninfected hepatocytes, infected hepatocytes and free virions, respectively. Uninfected hepatocytes are assumed to be produced by a constant rate $\Pi$, and die at the per capita rate of $\delta$. It becomes to infected hepatocytes at the rate of $\beta x v$, and $\beta$ is the infection rate constant which characterize the infection efficiency. The death rate of infected hepatocytes is by. Free virion are assumed to be produced from infected hepatocytes at the rate of $c y$, and $\gamma v$ is the clearance rate of viral particles. All parameters $\Pi, \delta, \beta, b, c$, and $\gamma$ are positive constants.

Mathematical analysis is an effective method to study the state of virus in vivo. According to the World Health Organization (WHO), over two billion people has been or is actively infected by $\mathrm{HBV}$ infections [14]. Chronic HBV infection often occurs in our early life and the virus persists in the body due to the destruction of strong antibody or cellular immune responses [15]. Recently, in an HBV model of viral infection, a reversion rate constant has been introduced into the uninfected state to reflect a non-cytolytic mechanism [16]. That is to say, the infected hepatocytes may be reverted to be uninfected state by losing all cccDNA from their nucleus [10]. Some research indicates that there is indeed a cytokine-mediated cure of infected cells during HBV infection [10,17-19]. Specifically, in [20] the authors considered the following model:

$$
\begin{aligned}
& \frac{\mathrm{d} x}{\mathrm{~d} t}=\Pi-\delta x-\beta x v+p y, \\
& \frac{\mathrm{d} y}{\mathrm{~d} t}=\beta x v-(b+p) y, \\
& \frac{\mathrm{d} v}{\mathrm{~d} t}=c y-\gamma v,
\end{aligned}
$$


TABLE 1: The biological meanings for state variables and parameters for system (3).

\begin{tabular}{|c|c|c|c|}
\hline Variables & Description & & \\
\hline$x$ & Number of the uninfected hepatocytes & & \\
\hline$y$ & Number of the infected hepatocytes & & \\
\hline$v$ & Number of the free virions & & \\
\hline Parameter & Description & Value & Units \\
\hline$\Pi$ & Recruitment rate & $5 \times 10^{5}$ & cells $\mathrm{ml}^{-1} \mathrm{~d}^{-1}$ \\
\hline$\delta$ & Natural death rate of uninfected hepatocytes & 0.003 & $\mathrm{~d}^{-1}$ \\
\hline$\beta$ & Infection rate & $4 \times 10^{-10}$ & $\mathrm{ml}$ copies $^{-1} \mathrm{~d}^{-1}$ \\
\hline$a$ & Saturation constant & 0.006 & $\mathrm{~d}^{-1}$ \\
\hline$p$ & Cure rate of infected hepatocytes & - & $d^{-1}$ \\
\hline$b$ & Death rate of infected hepatocytes & 0.1 & $d^{-1}$ \\
\hline$c$ & Number of free virions produced by infected hepatocytes & 6.24 & $d^{-1}$ \\
\hline$\gamma$ & Death rate of free virions & 0.65 & $\mathrm{~d}^{-1}$ \\
\hline$u_{1}$ & The drug effect on HBV by IFN & {$[0,1]$} & $d^{-1}$ \\
\hline$u_{2}$ & The drug effect on HBV by LAM & - & $d^{-1}$ \\
\hline
\end{tabular}

where the term $p y$ represents the rate at which infected hepatocytes are recovered to uninfected hepatocytes through cure.

As is known to all, differential equations provide an important tool for mathematical models, which are very useful in understanding the dynamic behavior of biological systems. However, many engineering, physical and biological systems have temporal memory [21-23]. Classical integerorder differential equations do not reflect this characteristic of memory. Therefore, it is necessary to introduce fractionalorder differential equations since fractional-order derivative can provide a useful instrument for description of memory and hereditary properties in many different domains. What's more, it was showed that fractional-order differential equations could simulate many phenomena that integer-order can not $[24,25]$. In fact, fractional calculus generalizes integrals and derivatives of integer-order to arbitrary orders [26-28]. It has been widely used in many different fields, such as finance [21], physics [22], biology [23], medicine [29] and so on.

Although bilinear incidence rate is mostly used in epidemic models, recently many literatures considered Holling type-II functional response as the incidence rate [2, 30-32]. In addition, our research is mainly to control the development of HBV. In fact, interferon (IFN) and lamivudine (LAM) are mainly used for treatment of HBV. They both have the effects of antivirus and antifibrosis. IFN is the most commonly used antiviral drug, it can inhibit virus' replication with extensive, indirect and species-specific. LAM is a cytosine nucleoside analogue that inhibits viral reverse transcriptase.

However, few literatures use fractional differential equations to describe the transmission of HBV with drug treatment. Therefore, based on the model (2), combining the fractional-order derivatives and the Holling type-II functional response, we propose the following improved $\mathrm{HBV}$ model with drug treatment:

$$
D^{\alpha} x=\Pi-\delta x-\left(1-u_{1}\right) \frac{\beta x v}{1+a x}+p y
$$

$$
\begin{aligned}
& D^{\alpha} y=\left(1-u_{1}\right) \frac{\beta x v}{1+a x}-(b+p) y, \\
& D^{\alpha} v=c y-\gamma v-u_{2} v
\end{aligned}
$$

where $u_{1} \in(0,1)$ and it represents the drug effect on HBV by IFN. $u_{2} \gg \gamma$ and it represents the drug effect on HBV by LAM. The biological meanings of state variables and parameters are shown in Table 1.

The organization of our current paper is as follows: In Section 2, we give some properties of fractional-order calculus. In Section 3, firstly, the existence and uniqueness of the positive solutions are proved; secondly, the sufficient conditions for the existence and the stability of two equilibriums for system (3) are obtained. In Section 4, some numerical simulations are performed to verify our theoretical results. Finally, this paper ends up with a brief discussion.

\section{Basic Properties of Fractional-Order Calculus}

In fractional-order calculus, we know there are many fractional-order integration and fractional-order differentiation that have been defined, for example, the GrunwaldLetnikov (GL) definition, the Riemann-Liouville (RL) definition and the Caputo definition. Since the initial conditions are in the same form as for the integer-order differential equations, we will adapt the Caputo's definition in our paper. Another advantage of this definition is that applied problems require definitions of fractional derivatives, where there are clear interpretations of initial conditions, which contain $f(a)$, $f^{\prime}(a), f^{\prime \prime}(a)$, etc.

Definition 1 (see [26]). The Caputo fractional-order derivative of a continuous function $f(x): \mathbf{R}^{+} \longrightarrow \mathbf{R}$ can be defined as

$$
\mathrm{D}^{\alpha} f(x)=\mathrm{I}^{n-\alpha} \mathrm{D}^{n} f(x), \quad \mathrm{D}=\frac{\mathrm{d}}{\mathrm{d} t},
$$

where $\alpha \in(n-1, n), n \in \mathbf{N}$. 
Lemma 2 (see [33], (generalized mean value theorem)). Assume that $f(t), D^{\alpha} f(t)$ are continuous functions on the interval $[a, b]$, then we have

$$
f(t)=f(a)+\frac{1}{\Gamma(\alpha)} \mathrm{D}^{\alpha} f(\xi)(t-a)^{\alpha},
$$

where $\xi \in[a, t]$.

Lemma 3 (see [33]). Assume that $f(t), \mathrm{D}^{\alpha} f(t)$ are continuous functions on the interval $[a, b]$, and $\alpha \in(0,1]$, then we have

(i) If $\mathrm{D}^{\alpha} f(t) \geq 0$, for $\forall t \in(a, b)$, then $f(t)$ is nondecreasing for each $t \in[a, b]$.

(ii) If $\mathrm{D}^{\alpha} f(t) \leq 0$, for $\forall t \in(a, b)$, then $f(t)$ is nonincreasing for each $t \in[a, b]$.

Lemma 4 (see [34]). Suppose that the vector function $f(t, X(t)): \mathbf{R}^{+} \times \mathbf{R}^{3} \longrightarrow \mathbf{R}^{3}$ satisfies the following conditions:

(i) Function $f(t, X(t))$ is Lebesgue measurable with respect to $t \in \mathbf{R}^{+}$;

(ii) Function $f(t, X(t))$ is continuous with respect to $X(t)$ on $\mathbf{R}^{3}$;

(iii) $\partial f(t, X) / \partial X$ is continuous with respect to $X(t)$ on $\mathbf{R}^{3}$;

(iv) $\|f(t, X)\| \leq \lambda\|X\|+\omega, \forall t \in \mathbf{R}^{+}, X \in \mathbf{R}^{3}$, where $\omega, \lambda$ are two positive constants.

Then the initial value problems (IVP)

$$
\begin{aligned}
\mathrm{D}^{\alpha} X(t) & =f(t, X(t)), \quad \alpha \in(0,1], \\
X\left(t_{0}\right) & =X_{0},
\end{aligned}
$$

has a unique solution.

\section{Analysis of System (3)}

In this section, firstly, the existence and uniqueness of positive solution is proved; secondly, the basic reproduction number and the existence conditions for both equilibriums (diseasefree equilibrium and endemic equilibrium) are obtained; lastly, the conditions for the stability of both equilibriums are obtained.

\subsection{The Existence and Uniqueness of Positive Solutions}

Theorem 5. System (3) with any positive initial value has a unique solution, and it remains within $\Omega$, where

$$
\begin{aligned}
\Omega & =\left\{(x, y, v) \in \mathbf{R}_{+}^{3}: 0 \leq x+y \leq \frac{\Pi}{\delta}, 0 \leq v\right. \\
& \left.\leq \frac{c \Pi}{\delta\left(\gamma+u_{2}\right)}\right\} .
\end{aligned}
$$

Proof. We will accomplish the proof through three steps:

Step 1. In this step, we will show that system (3) with any positive initial value has a unique solution.
Denote the right side of system (3) as vector function $f(t, X(t))$, then the corresponding conditions (i)-(iii) of Lemma 4 are satisfied. So, we only need to prove that system (3) satisfies the fourth condition of Lemma 4.

$$
\text { Let } x_{1}(t)=x(t), x_{2}(t)=y(t), x_{3}(t)=v(t), x_{1}(0)=x(0)=
$$$$
x_{0}, x_{2}(0)=y(0)=y_{0}, x_{3}(0)=v(0)=v_{0} \text {, and }
$$

$$
\begin{aligned}
X(t) & =\left(\begin{array}{l}
x_{1}(t) \\
x_{2}(t) \\
x_{3}(t)
\end{array}\right), \\
\eta & =\left(\begin{array}{l}
\Pi \\
0 \\
0
\end{array}\right), \\
A_{1} & =\left(\begin{array}{ccc}
-\delta & p & 0 \\
0 & -(b+p) & 0 \\
0 & -\gamma-u_{2}
\end{array}\right), \\
A_{2} & =\left(\begin{array}{ccc}
0 & 0 & -\frac{\beta\left(1-u_{1}\right)}{a} \\
0 & 0 & \frac{\beta\left(1-u_{1}\right)}{a} \\
0 & 0 & 0
\end{array}\right),
\end{aligned}
$$

then system (3) can be reduced to the following form:

$$
\mathrm{D}^{\alpha} X(t)=A_{1} X(t)+\frac{x_{1}(t)}{1 / a+x_{1}(t)} A_{2} X(t)+\eta .
$$

Denote

$$
f(t, X(t))=A_{1} X(t)+\frac{x_{1}(t)}{1 / a+x_{1}(t)} A_{2} X(t)+\eta,
$$

and then

$$
\begin{aligned}
\|f(t, X(t))\| & =\left\|A_{1} X(t)+\frac{x_{1}(t)}{1 / a+x_{1}(t)} A_{2} X(t)+\eta\right\| \\
& \leq\left\|A_{1}\right\| \cdot\|X(t)\|+\left\|A_{2}\right\| \cdot\|X(t)\|+\|\eta\| \\
& =\left(\left\|A_{1}\right\|+\left\|A_{2}\right\|\right)\|X(t)\|+\|\eta\| \\
& \doteq \lambda\|X(t)\|+\omega .
\end{aligned}
$$

According to Lemma 4, system (3) with any positive initial values has a unique solution.

Step 2. We will prove that the solution of system (3) with positive initial values is always non-negative. From system (3), we easily get

$$
\begin{aligned}
& \left.\mathrm{D}^{\alpha} x\right|_{x=0}=\Pi+p y \geq 0, \\
& \left.\mathrm{D}^{\alpha} y\right|_{y=0}=\frac{\beta\left(1-u_{1}\right) x v}{1+a x} \geq 0, \\
& \left.\mathrm{D}^{\alpha} v\right|_{v=0}=c y \geq 0 .
\end{aligned}
$$

From Lemma 3, we have $x(t), y(t), v(t) \geq 0$ for any $t \geq 0$. As a result, the solution of system (3) will remain in $\mathbf{R}_{+}^{3}$. 
Step 3. Assume that $\delta \leq b$ for the biological justification. Adding the first two equations of system (3), we have

$$
\mathrm{D}^{\alpha}(x+y)=\Pi-\delta x-b y \leq \Pi-\delta(x+y),
$$

which implies that

$$
x(t)+y(t) \leq\left[-\frac{\Pi}{\delta}+x(0)+y(0)\right] E_{\alpha}\left(-\delta t^{\alpha}\right)+\frac{\Pi}{\delta} .
$$

Since $E_{\alpha}\left(-\delta t^{\alpha}\right) \geq 0$ for any $t \geq 0$, then we have

$$
x(t)+y(t) \leq \frac{\Pi}{\delta}, \quad \forall t \geq 0,
$$

provided that $x(0)+y(0) \leq \Pi / \delta$.

From the last equation of system (3) we get

$$
\mathrm{D}^{\alpha} v \leq \frac{c \Pi}{\delta}-\gamma v-u_{2} v
$$

and from which we get that $v(t) \leq c \Pi / \delta\left(\gamma+u_{2}\right)$ as time $t$ large enough.

From the results of step 2 and step 3, we know that $\Omega$ is a positive invariant set with respect to system (3).

Since $\Omega$ is positive invariant with respect to system (3), we only need to consider this system within $\Omega$ in the rest of this section.

3.2. Basic Reproduction Number and the Existence of Both Equilibriums. In the process of studying disease transmission, what we most concerned about is how to eradicate or control the disease within a range. Many epidemiological models have a disease-free equilibrium where the disease is not present in the population. Therefore, we need a threshold to estimate whether the disease is present or not in the population. This threshold is called basic reproduction number. By the next generation matrix approach given in [35], we can get the basic reproduction number of system (3) as follows:

$$
R_{u}=\rho\left(F_{u} V_{u}^{-1}\right)=\frac{c \beta \Pi\left(1-u_{1}\right)}{\left(\gamma+u_{2}\right)(b+p)(\delta+a \Pi)},
$$

where

$$
\begin{aligned}
F_{u} & =\left(\begin{array}{cc}
0 & \left(1-u_{1}\right) \frac{\beta \Pi}{\delta+a \Pi} \\
0 & 0
\end{array}\right), \\
V_{u} & =\left(\begin{array}{cc}
b+p & 0 \\
-c & \gamma+u_{2}
\end{array}\right) .
\end{aligned}
$$

Corollary 6. (1) When $u_{1}=u_{2}=0$, we obtain $R_{u}=$ $c \beta \Pi / \gamma(b+p)(\delta+a \Pi) \doteq R_{0}$. Here $R_{0}$ is called basic reproduction number, and it is a threshold to estimate whether the disease is persistent or not.

(2) In addition, through the eigenvalue analysis method, we can easily prove that the disease-free equilibrium is stable when $R_{0}<1$; and it is unstable when $R_{0}>1$.
Let the right side of system (3) equal to zero, we obtain an algebraic equations as follows

$$
\begin{aligned}
\Pi-\delta x-\left(1-u_{1}\right) \frac{\beta x v}{1+a x}+p y & =0, \\
\left(1-u_{1}\right) \frac{\beta x v}{1+a x}-(b+p) y & =0, \\
c y-\gamma v-u_{2} v & =0 .
\end{aligned}
$$

By simple calculation, we obtain two equilibriums of the system (3), namely:

(i) There always exists a disease-free equilibrium $E_{u}^{0}=$ $(\Pi / \delta, 0,0)$;

(ii) If $R_{u}>1$, then there exists a unique endemic equilibrium $E_{u}^{*}=\left(x^{*}, y^{*}, v^{*}\right)$, where

$$
\begin{aligned}
x^{*} & =\frac{\left(\gamma+u_{2}\right)(b+p)}{c \beta\left(1-u_{1}\right)-a\left(\gamma+u_{2}\right)(b+p)}, \\
y^{*} & =\frac{c \beta \Pi\left(1-u_{1}\right)}{b\left[c \beta\left(1-u_{1}\right)-a\left(\gamma+u_{2}\right)(b+p)\right]}\left[1-\frac{1}{R_{u}}\right], \\
v^{*} & =\frac{c^{2} \beta \Pi\left(1-u_{1}\right)}{b\left(\gamma+u_{2}\right)\left[c \beta\left(1-u_{1}\right)-a\left(\gamma+u_{2}\right)(b+p)\right]}[1 \\
& \left.-\frac{1}{R_{u}}\right] .
\end{aligned}
$$

\subsection{Stability Analysis of the Two Equilibriums}

Theorem 7. The disease-free equilibrium $E_{u}^{0}$ is locally asymptotically stable if $R_{u}<1$, and it is unstable if the inequality is reversed.

Proof. The Jacobian matrix evaluated at the disease-free equilibrium $E_{u}^{0}$ is given by

$$
J\left(E_{u}^{0}\right)=\left(\begin{array}{ccc}
-\delta & p & -\left(1-u_{1}\right) \frac{\beta \Pi}{\delta+a \Pi} \\
0 & -(b+p) & \left(1-u_{1}\right) \frac{\beta \Pi}{\delta+a \Pi} \\
0 & c & -\gamma-u_{2}
\end{array}\right),
$$

and the corresponding characteristic equation at the diseasefree equilibrium $E_{u}^{0}$ is

$$
\left|\begin{array}{ccc}
\lambda+\delta & -p & \frac{\beta \Pi}{\delta+a \Pi} \\
0 & \lambda+(b+p) & -\frac{\beta \Pi}{\delta+a \Pi} \\
0 & -c & \lambda+\gamma
\end{array}\right|=0 .
$$

It is easily seen that one of the eigenvalues of (22) is $\lambda_{1}=$ $-\delta<0$, and the other two eigenvalues are determined by the following quadratic equation:

$$
\lambda^{2}+d_{1} \lambda+d_{2}=0
$$

where

$$
\begin{aligned}
& d_{1}=b+p+\gamma+u_{2}>0, \\
& d_{2}=\left(\gamma+u_{2}\right)(b+p)\left(1-R_{u}\right) .
\end{aligned}
$$


If $R_{u}<1$, then $d_{2}>0$ and both roots of (23) have negative real part. Thus, the disease-free equilibrium $E_{u}^{0}$ is locally asymptotically stable.

If $R_{u}>1$, then $d_{2}<0$, which means that one root of (23) is positive. Thus, the disease-free equilibrium $E_{u}^{0}$ is unstable.

Remark 8 . When $u_{1}=u_{2}=0$, if the parameters satisfy $R_{u}=R_{0}<1$, then the disease-free equilibrium is locally asymptotically stable, which means no drug treatments are employed.

Remark 9. If the parameters satisfy $R_{0}>1$, then the diseasefree equilibrium will be unstable if there is no drug treatment. We can employ suitable drug treatment to control the disease, specifically we have the following:
(1) If $u_{1}=0, u_{2} \neq 0$, which means only LAM is employed to treat $\mathrm{HBV}$, then we can select suitable $u_{2}$ such that $R_{u}<$ $1<R_{0}$. According to Theorem 7, $E_{u}^{0}$ is locally asymptotically stable.

(2) If $u_{1} \neq 0, u_{2}=0$, which means only IFN is employed to treat $\mathrm{HBV}$, then we can select suitable $u_{1}$ such that $R_{u}<$ $1<R_{0}$. According to Theorem $7, E_{u}^{0}$ is locally asymptotically stable.

(3) If $u_{1} \neq 0$ and $u_{2} \neq 0$, which means two drugs are used to treat HBV, then we can select suitable $u_{1}$ and $u_{2}$ such that $R_{u}<1<R_{0}$. According to Theorem 7, $E_{u}^{0}$ is locally asymptotically stable.

To discuss the local stability of the endemic equilibrium $E_{u}^{*}$, we consider the linearized system of (3) at $E_{u}^{*}$. The Jacobian matrix at the endemic equilibrium $E_{u}^{*}$ is given by:

$$
J\left(E_{u}^{*}\right)=\left(\begin{array}{ccc}
-\delta-\frac{\Pi\left[c \beta\left(1-u_{1}\right)-a\left(\gamma+u_{2}\right)(b+p)\right]}{b\left(\gamma+u_{2}\right)}\left[1-\frac{1}{R_{0} u}\right] & p & -\frac{\left(\gamma+u_{2}\right)(b+p)}{c} \\
\frac{\Pi\left[c \beta\left(1-u_{1}\right)-a\left(\gamma+u_{2}\right)(b+p)\right]}{b\left(\gamma+u_{2}\right)}\left[1-\frac{1}{R_{u}}\right] & -(b+p) & \frac{\left(\gamma+u_{2}\right)(b+p)}{c} \\
0 & c & -\left(\gamma+u_{2}\right)
\end{array}\right) .
$$

The corresponding characteristic equation at the endemic equilibrium $E_{u}^{*}$ is

$$
P(\mu)=\mu^{3}+\kappa_{1} \mu^{2}+\kappa_{2} \mu+\kappa_{3}=0,
$$

where

$$
\begin{aligned}
\kappa_{1}= & \left(\delta+b+p+\gamma+u_{2}\right) \\
& +\frac{\Pi\left[c \beta\left(1-u_{1}\right)-a\left(\gamma+u_{2}\right)(b+p)\right]}{b\left(\gamma+u_{2}\right)}\left[1-\frac{1}{R_{u}}\right], \\
\kappa_{2}= & \delta\left(b+p+\gamma+u_{2}\right)+\left(b+\gamma+u_{2}\right) \\
& . \frac{\Pi\left[c \beta\left(1-u_{1}\right)-a\left(\gamma+u_{2}\right)(b+p)\right]}{b\left(\gamma+u_{2}\right)}\left[1-\frac{1}{R_{u}}\right], \\
\kappa_{3}= & \Pi\left[c \beta\left(1-u_{1}\right)-a\left(\gamma+u_{2}\right)(b+p)\right]\left[1-\frac{1}{R_{u}}\right] .
\end{aligned}
$$

Proposition 10. The endemic equilibrium $E_{u}^{*}$ is locally asymptotic stable if all of the eigenvalues $\mu_{i}$ of Eq.(26) satisfy $\left|\arg \left(\mu_{i}\right)\right|>\alpha \pi / 2, i=1,2,3$.

Denote $D(P)$ as the discriminant of $P(\mu)$, where

$$
\begin{aligned}
D(P) & =-\left|\begin{array}{ccccc}
1 & \kappa_{1} & \kappa_{2} & \kappa_{3} & 0 \\
0 & 1 & \kappa_{1} & \kappa_{2} & \kappa_{3} \\
3 & 2 \kappa_{1} & \kappa_{2} & 0 & 0 \\
0 & 3 & 2 \kappa_{1} & \kappa_{2} & 0 \\
0 & 0 & 3 & 2 \kappa_{1} & \kappa_{2}
\end{array}\right| \\
& =18 \kappa_{1} \kappa_{2} \kappa_{3}+\left(\kappa_{1} \kappa_{2}\right)^{2}-4 \kappa_{1}^{3} \kappa_{3}-4 \kappa_{2}^{3}-27 \kappa_{3}^{2} .
\end{aligned}
$$

In order to discuss the stability of the endemic equilibrium $E_{u}^{*}$, we get the following result by use of the same method as in [36].

Proposition 11. (i) If the discriminant $D(P)>0$, then the endemic equilibrium $E_{u}^{*}$ is locally asymptotically stable if and only if the Routh-Hurwitz conditions are satisfied; that is to say, $\kappa_{1}>0, \kappa_{3}>0, \kappa_{1} \kappa_{2}>\kappa_{3}$.

(ii) If the discriminant $D(P)<0, \kappa_{1}>0, \kappa_{2}>0, \kappa_{1} \kappa_{2}=\kappa_{3}$, and $\alpha \in(0.5,1)$, then the endemic equilibrium $E_{u}^{*}$ is locally asymptotically stable.

(iii) If the discriminant $D(P)<0, \kappa_{1} \geq 0, \kappa_{2} \geq 0, \kappa_{3}>0$, $0.5<\alpha<2 / 3$, then the endemic equilibrium $E_{u}^{*}$ is locally asymptotically stable.

(iv) If the discriminant $D(P)<0, \kappa_{1}<0, \kappa_{2}<0, \alpha>2 / 3$, then the endemic equilibrium $E_{u}^{*}$ is unstable.

About the global stability of the disease-free equilibrium $E_{u}^{0}$, we have the following result.

Theorem 12. The disease-free equilibrium $E_{u}^{0}$ is globally asymptotically stable if $R_{u} \leq 1$.

Proof. Consider the following Lyapunov function

$$
L=y+\frac{\left(1-u_{1}\right) \beta x_{0}}{\left(\gamma+u_{2}\right)\left(1+a x_{0}\right)} v, \quad x_{0}=\frac{\Pi}{\delta} .
$$

The derivative of $L$ along a solution of system (3) is:

$$
\left.\mathrm{D}^{\alpha} L\right|_{(3)}=\mathrm{D}^{\alpha} y+\frac{\left(1-u_{1}\right) \beta x_{0}}{\left(\gamma+u_{2}\right)\left(1+a x_{0}\right)} \mathrm{D}^{\alpha} v
$$




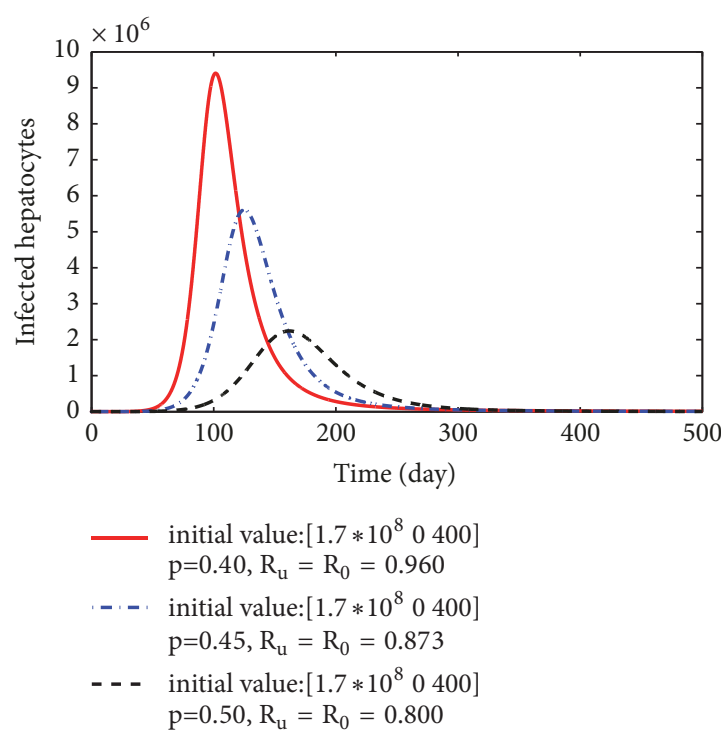

(a) $\mathrm{u}_{1}=\mathrm{u}_{2}=0, \alpha=0.9$

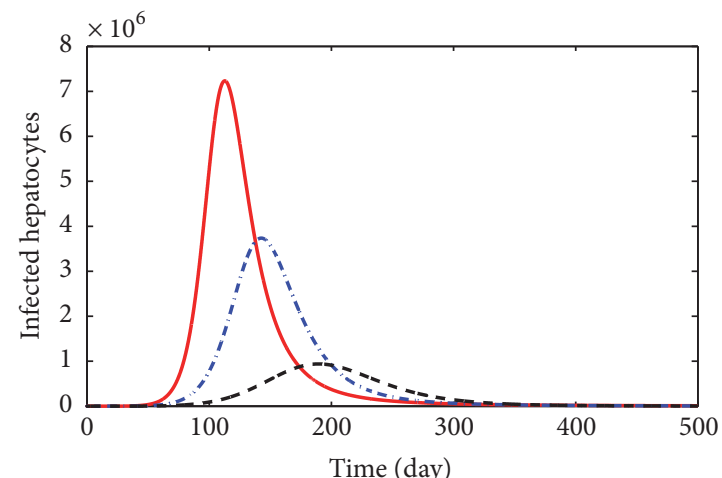
initial value:[1.5*10 $0^{8} 0300$ ] $\mathrm{p}=0.40, \mathrm{R}_{\mathrm{u}}=\mathrm{R}_{0}=0.960$
. _. - initial value: $\left[1.5 * 10^{8} 0300\right]$ $\mathrm{p}=0.45, \mathrm{R}_{\mathrm{u}}=\mathrm{R}_{0}=0.873$
- - - initial value: $\left[1.5 * 10^{8} 0300\right]$ $\mathrm{p}=0.50, \mathrm{R}_{\mathrm{u}}=\mathrm{R}_{0}=0.800$

(c) $\mathrm{u}_{1}=\mathrm{u}_{2}=0, \alpha=0.90$

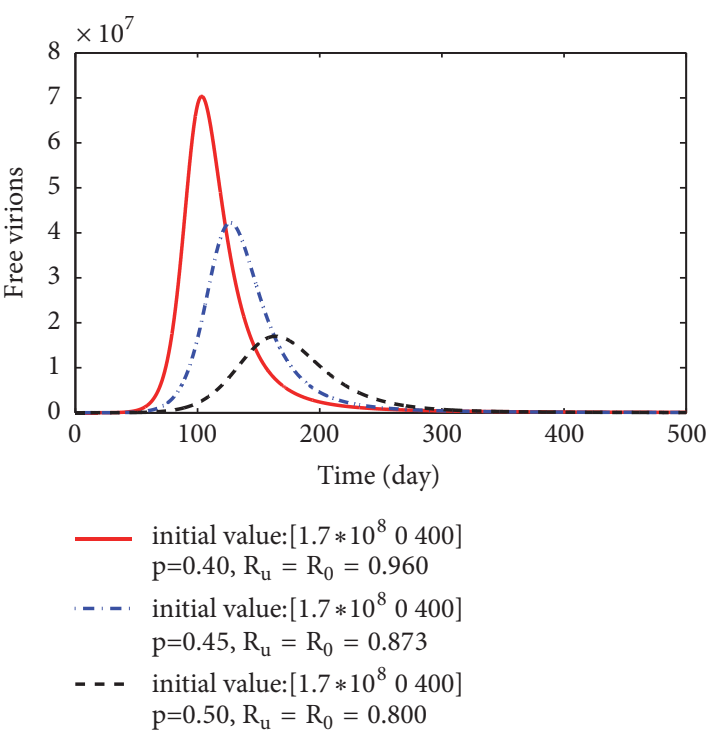

(b) $\mathrm{u}_{1}=\mathrm{u}_{2}=0, \alpha=0.90$

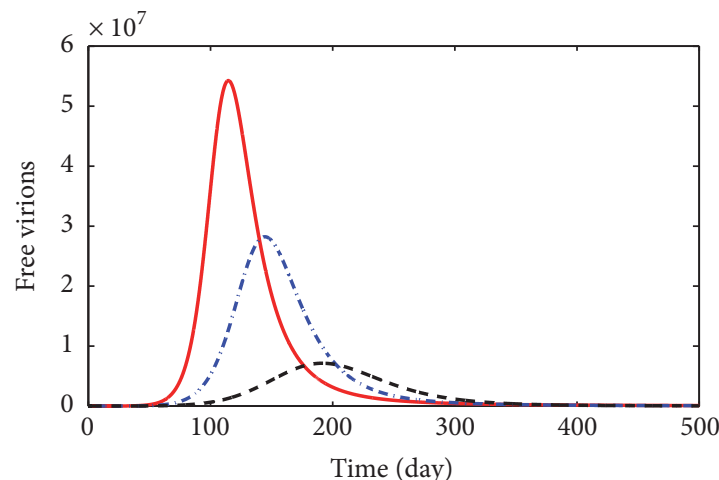
initial value: $\left[1.5 * 10^{8} 0300\right]$ $\mathrm{p}=0.40, \mathrm{R}_{\mathrm{u}}=\mathrm{R}_{0}=0.960$
. - - initial value: $\left[1.5 * 10^{8} 0300\right]$ $\mathrm{p}=0.45, \mathrm{R}_{\mathrm{u}}=\mathrm{R}_{0}=0.873$
- - - initial value: $\left[1.5 * 10^{8} 0300\right]$ $\mathrm{p}=0.50, \mathrm{R}_{\mathrm{u}}=\mathrm{R}_{0}=0.800$

(d) $\mathrm{u}_{1}=\mathrm{u}_{2}=0, \alpha=0.90$

Figure 1: Dynamics of system (3) for different values of $p$, which shows that the disease-free equilibrium $E_{u}^{0}=(\Pi / \delta, 0,0)=\left(1.667 \times 10^{8}, 0,0\right)$

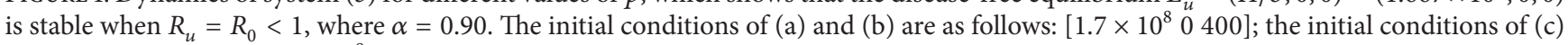
and (d) are as follows: [1.5 $\left.\times 10^{8} 0300\right]$.

$$
\begin{aligned}
= & \frac{\left(1-u_{1}\right) \beta x v}{1+a x}-(b+p) y \\
& +\frac{\left(1-u_{1}\right) \beta x_{0}}{\left(\gamma+u_{2}\right)\left(1+a x_{0}\right)}\left(c y-\gamma v-u_{2} v\right) \\
= & \left(1-u_{1}\right) \beta\left[\frac{x}{1+a x}-\frac{x_{0}}{1+a x_{0}}\right] v \\
& +\left[\frac{\left(1-u_{1}\right) c \beta x_{0}}{\left(\gamma+u_{2}\right)\left(1+a x_{0}\right)}-(b+p)\right] y
\end{aligned}
$$

$$
\begin{aligned}
= & \left(1-u_{1}\right) \beta\left[\frac{x-x_{0}}{(1+a x)\left(1+a x_{0}\right)}\right] v \\
& +(b+p)\left(R_{u}-1\right) y \leq(b+p)\left(R_{u}-1\right) y,
\end{aligned}
$$

and the last inequality is obtained since $x(t) \leq x_{0}$.

If $R_{u} \leq 1$, then we have $\left.\mathrm{D}^{\alpha} L\right|_{(3)} \leq 0$. In addition, we know that the maximum invariant set for $\left\{(x, y, v) \in \Omega:\left.\mathrm{D}^{\alpha} L\right|_{(3)}=\right.$ $0\}$ is singleton $\left\{E_{u}^{0}\right\}$. According to the LaSalle's invariance principle, we know that all solutions in $\Omega$ converge to $E_{u}^{0}$.

This completes the proof. 


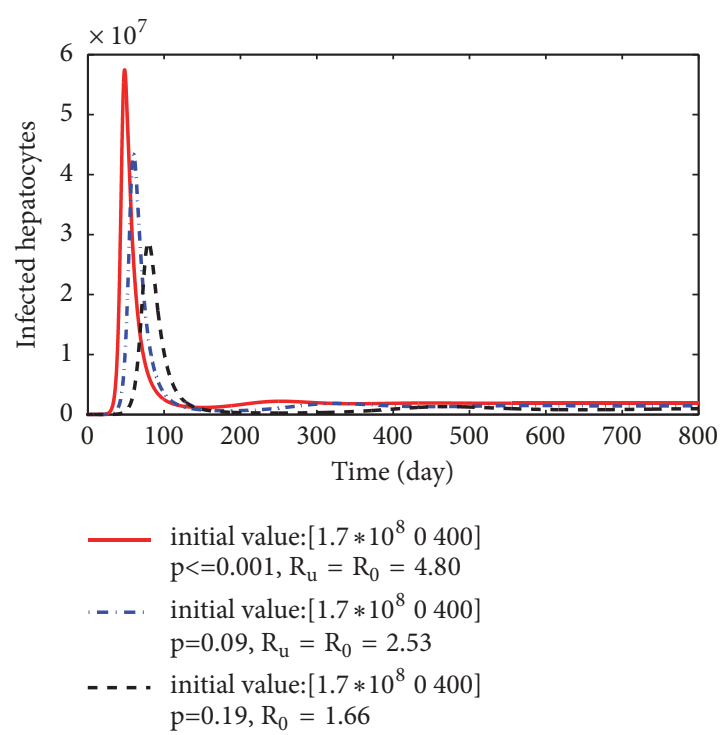

(a) $\mathrm{u}_{1}=\mathrm{u}_{2}=0, \alpha=0.95$

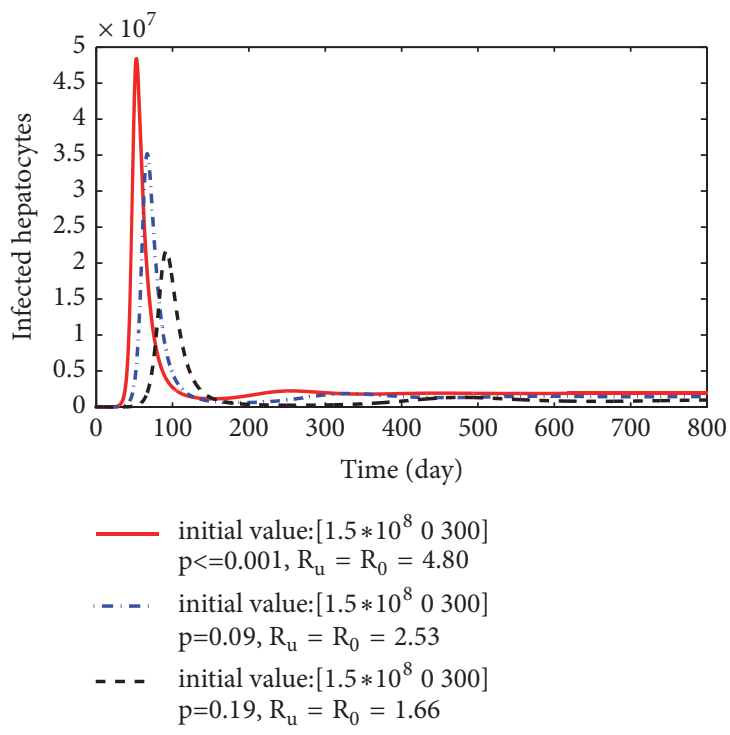

(c) $\mathrm{u}_{1}=\mathrm{u}_{2}=0, \alpha=0.95$

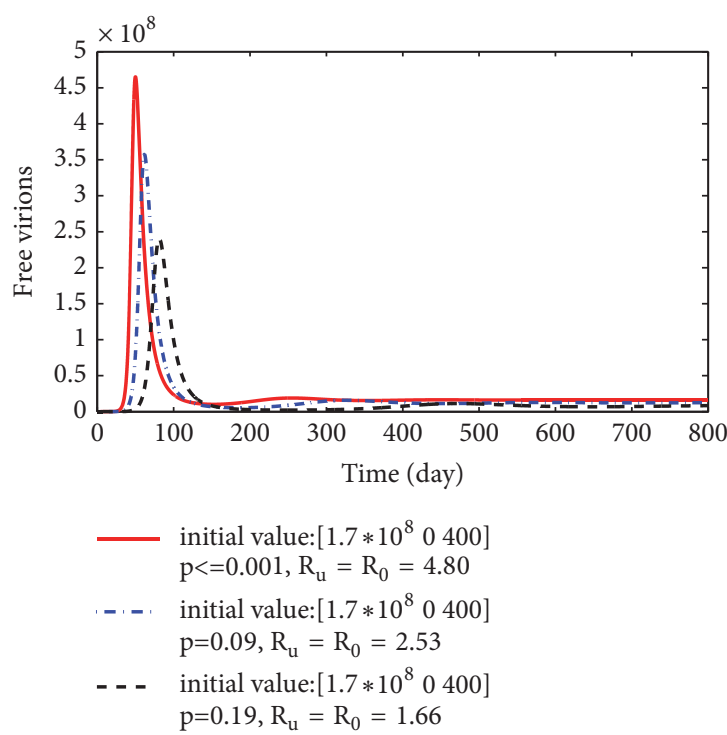

(b) $\mathrm{u}_{1}=\mathrm{u}_{2}=0, \alpha=0.95$

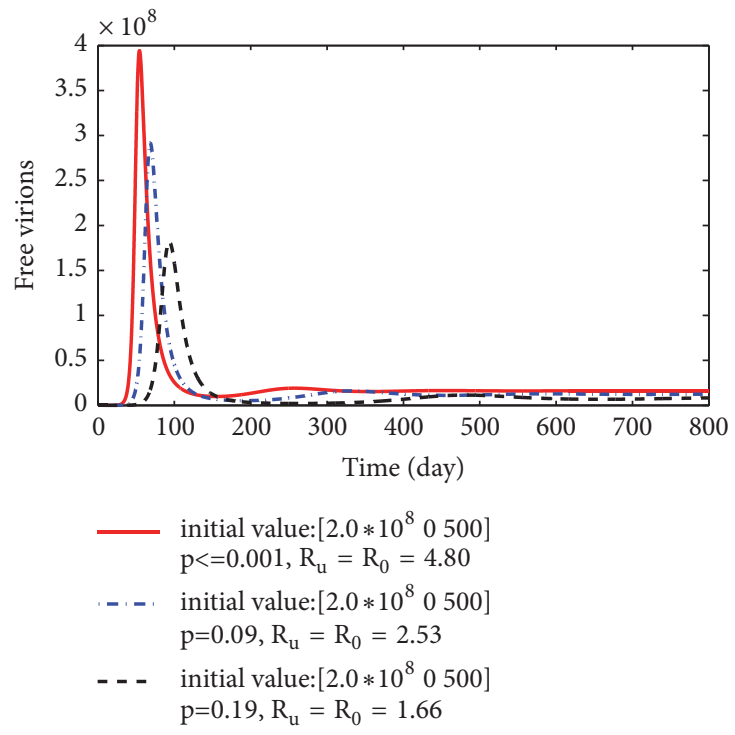

(d) $\mathrm{u}_{1}=\mathrm{u}_{2}=0, \alpha=0.95$

FIGURE 2: Dynamics of system (3) for different values of $p$, which shows that the disease-free equilibrium $E_{u}^{0}=(\Pi / \delta, 0,0)=\left(1.667 \times 10^{8}, 0,0\right)$ is unstable when $R_{u}=R_{0}>1$, where $\alpha=0.95$. The initial conditions of (a) and (b) are as follows: $\left[1.7 \times 10^{8} 0400\right]$; the initial conditions of (c) and (d) are as follows: $\left[1.5 \times 10^{8} 0300\right]$.

\section{Numerical Simulations for System (3)}

In previous sections, we have predicted some dynamical behavior of system (3). In this section, we will explore some numerical simulations to verify our theoretical results. The values of parameters are given in Table 1, and most of the values are taken from [37]. Since we are only concerned with the number of infected hepatocytes and the concentration of free virions, only $y(t)$ and $v(t)$ are shown in our illustration.

Figure 1 shows that when $\alpha=0.90$, if the value of $p$ is relatively big, then $R_{u}=R_{0}<1$, and the disease-free equilibrium $E_{u}^{0}$ is locally asymptotically stable.
Figure 2 shows that when $\alpha=0.95$, if the value of $p$ is relatively small, then $R_{u}=R_{0}>1$, and the disease-free equilibrium $E_{u}^{0}$ is unstable.

Remark 13. From Figures 1 and 2, we find that the value of cure rate $p$ is very important for the dynamics of the system. If the value of $p$ is relatively small, the disease will persist; while the disease will eradicate if the value of $p$ is relatively big. In addition, we also find that the initial values are not sensitive to the dynamical behaviors.

Figure 3 shows that if $u_{1}=0, u_{2} \neq 0$, as long as $R_{u}<1$, then the disease-free equilibrium $E_{u}^{0}$ is stable. 


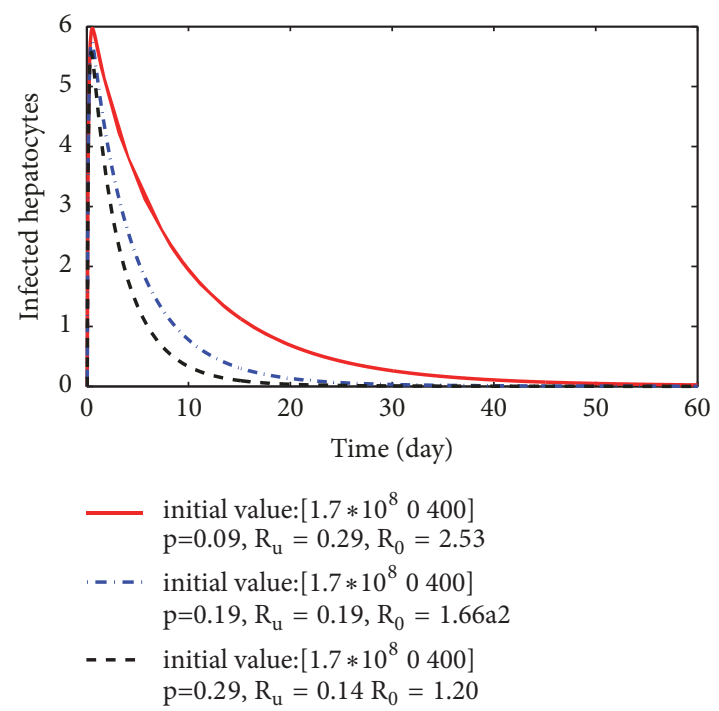

(a) $\mathrm{u}_{1}=0, \mathrm{u}_{2}=5, \alpha=0.95$

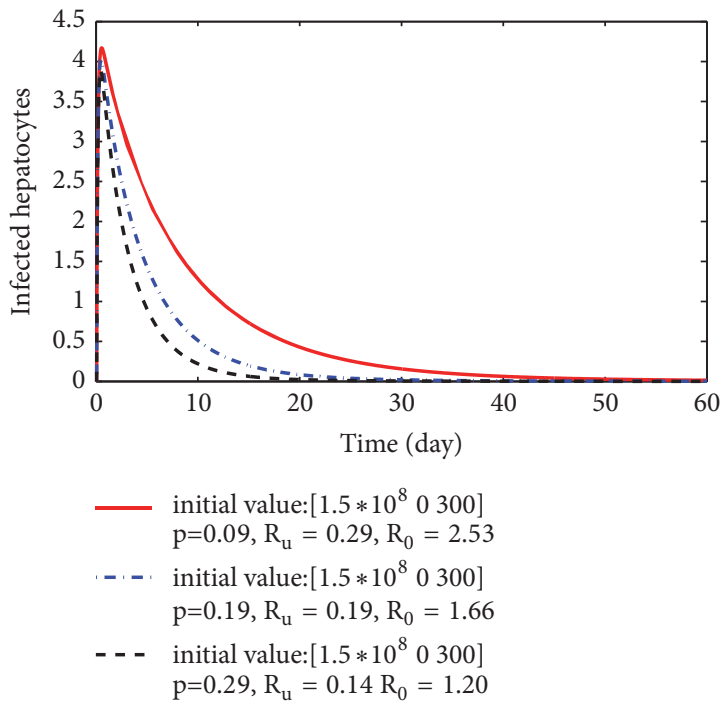

(c) $\mathrm{u}_{1}=0, \mathrm{u}_{2}=5, \alpha=0.95$

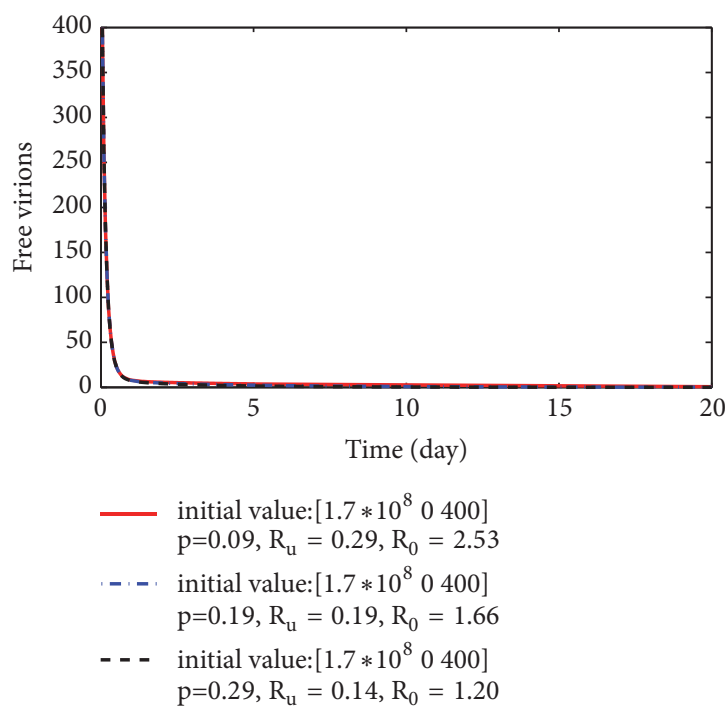

(b) $\mathrm{u}_{1}=0, \mathrm{u}_{2}=5, \alpha=0.95$

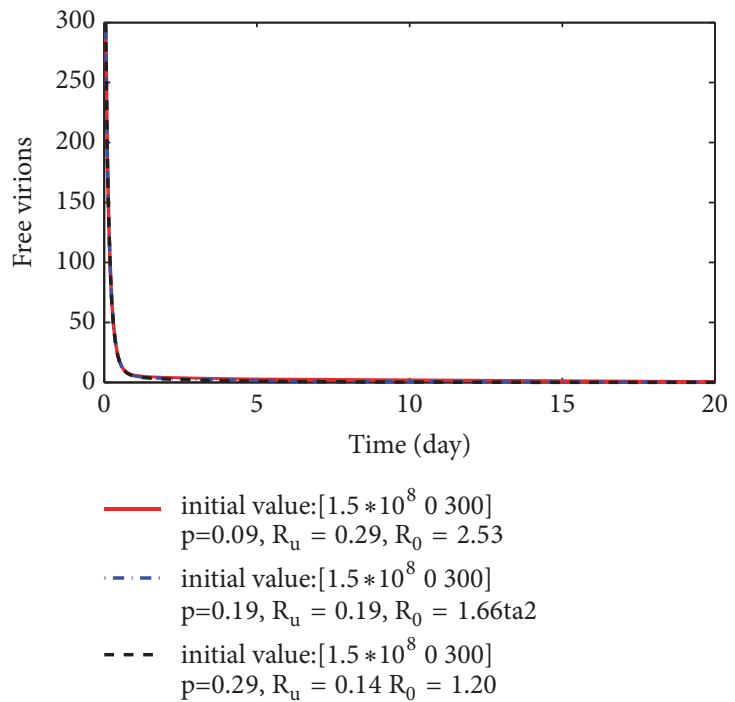

(d) $\mathrm{u}_{1}=0, \mathrm{u}_{2}=5, \alpha=0.95$

FIGURE 3: In this case, only LAM(without IFN) is used to treat HBV. For different values of $p$, when $R_{u}<1<R_{0}$, the disease-free equilibrium $E_{u}^{0}=(\Pi / \delta, 0,0)=\left(1.667 \times 10^{8}, 0,0\right)$ is asymptotically stable.The initial conditions of (a) and (b) are as follows: [1.7 $\left.\times 10^{8} 0400\right]$; the initial conditions of $(\mathrm{c})$ and $(\mathrm{d})$ are as follows: $\left[1.5 \times 10^{8} 0300\right]$.

Figure 4 shows that if $u_{1} \neq 0, u_{2}=0$, as long as $R_{u}<1$, then the disease-free equilibrium $E_{u}^{0}$ is stable.

Figure 5 shows that if $u_{1} \neq 0, u_{2} \neq 0$, as long as $R_{u}<1$, then the disease-free equilibrium $E_{u}^{0}$ is stable.

Remark 14. From these three figures, we found that whatever the initial values are, if we choose suitable values of $u_{1}$ and/or $u_{2}$ such that $R_{u}<1<R_{0}$, then the disease-free equilibrium $E_{u}^{0}$ is stable. The numerical simulation results are consistent with the Theorem 7 and Remark 9. That is to say, $R_{u}$ is a threshold to determine whether the disease is persistent or not.

From (a)-(b) of Figure 6 we find that the disease-free equilibrium $E_{u}^{0}$ is always asymptotically stable. At this case, only LAM (without IFN) is used to treat HBV.
From (c)-(d) of Figure 6 we find that the disease-free equilibrium $E_{u}^{0}$ is always asymptotically stable. At this case, only IFN (without LAM) is used to control HBV.

From (e)-(f) of Figure 6 we find that the disease-free equilibrium $E_{u}^{0}$ is always asymptotically stable. At this case, both IFN and LAM are used to control HBV.

Remark 15. (1) From Figure 6, we can see that no matter one or two control measures are taken, the disease-free equilibrium $E_{u}^{0}$ is always stable, as long as the values of $u_{1}$ and $u_{2}$ satisfy $R_{u}<1<R_{0}$.

(2) From Figures 6(a) and 6(e), we can see that as the value of $\alpha$ increases, the peak of disease infection decreases; from Figure $6(\mathrm{c})$, we can see that the peak of disease infection increases as the value of $\alpha$ increases. These simulation results 


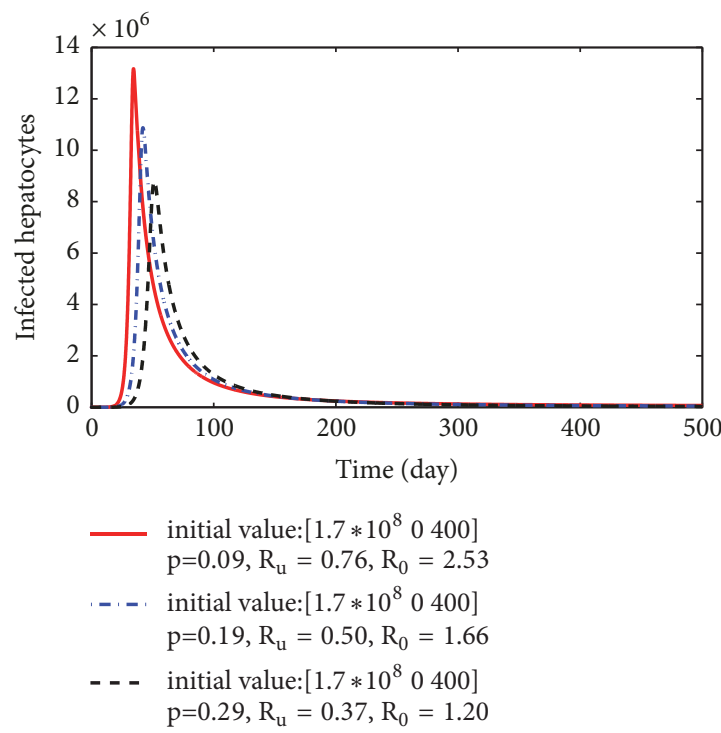

(a) $\mathrm{u}_{1}=0.7, \mathrm{u}_{2}=0, \alpha=0.95$

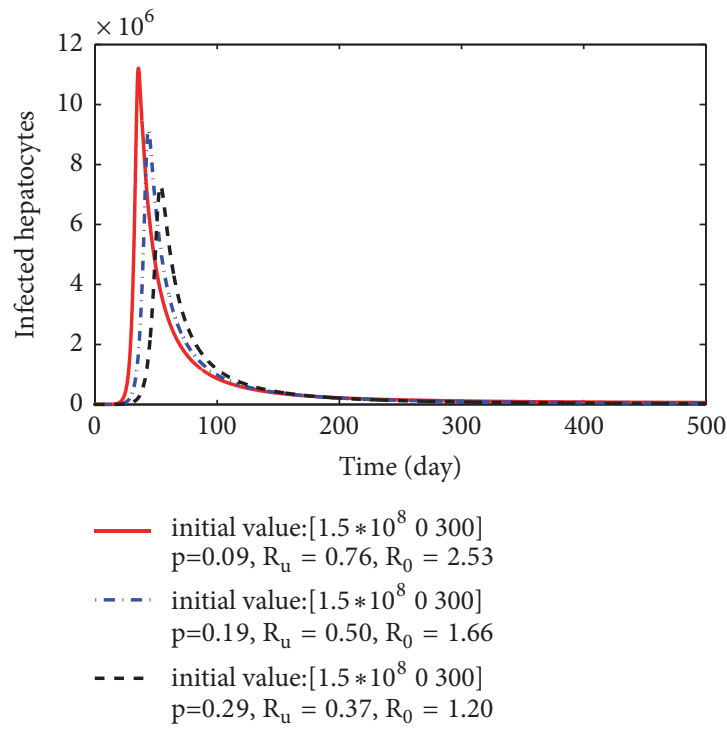

(c) $\mathrm{u}_{1}=0.7, \mathrm{u}_{2}=0, \alpha=0.95$

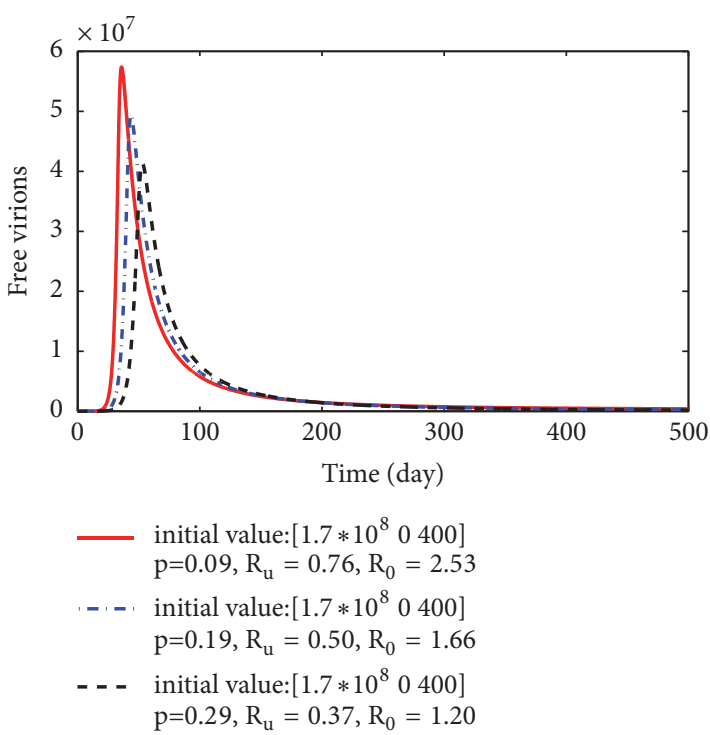

(b) $\mathrm{u}_{1}=0.7, \mathrm{u}_{2}=0, \alpha=0.95$

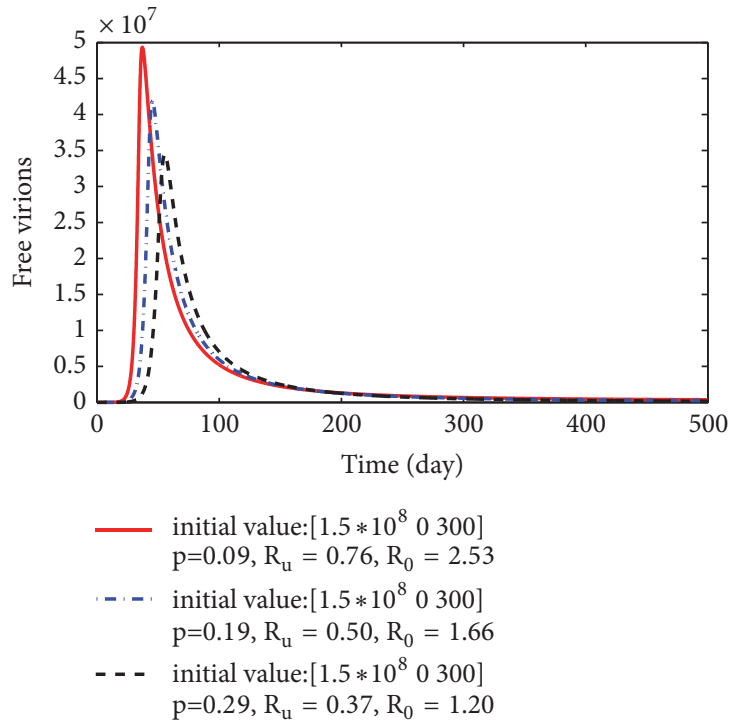

(d) $\mathrm{u}_{1}=0.7, \mathrm{u}_{2}=0, \alpha=0.95$

FIGURE 4: In this case, only IFN(without LAM) is used to treat HBV. For different values of $p$, when $R_{u}<1<R_{0}$, the disease-free equilibrium $E_{u}^{0}=(\Pi / \delta, 0,0)=\left(1.667 \times 10^{8}, 0,0\right)$ is asymptotically stable. The initial conditions of (a) and (b) are as follows: $\left[1.7 \times 10^{8} 0400\right]$; the initial conditions of $(\mathrm{c})$ and $(\mathrm{d})$ are as follows: $\left[1.5 \times 10^{8} 0300\right]$.

indicate that the value of $\alpha$ is an important factor for the peak of disease infection.

From Figures 7(a) and 7(b) we find that when $\alpha=1, R_{u}>$ 1 and the endemic equilibrium $E_{u}^{*}$ is always stable for three different initial values.

From Figures 7(c) and 7(d) we find that when $\alpha=0.5$, although $R_{u}>1$, the endemic equilibrium $E_{u}^{*}$ is unstable for three different initial values.

Remark 16. From Figure 7, we find that under the condition of $R_{u}>1$, the endemic equilibrium $E_{u}^{*}$ may be stable or unstable for different values of $\alpha$.

\section{Discussion}

In this article, we construct a fractional-order model with Holling II functional response to describe the transmission of HBV. We do the following work:

(1) We proved the existence and uniqueness of positive solutions.

(2) The basic reproduction number and the sufficient conditions for the existence of two equilibriums are obtained.

(3) We analyzed the stability of equilibriums are analyzed.

(4) Some numerical simulations are performed to verify our theoretical prediction. 


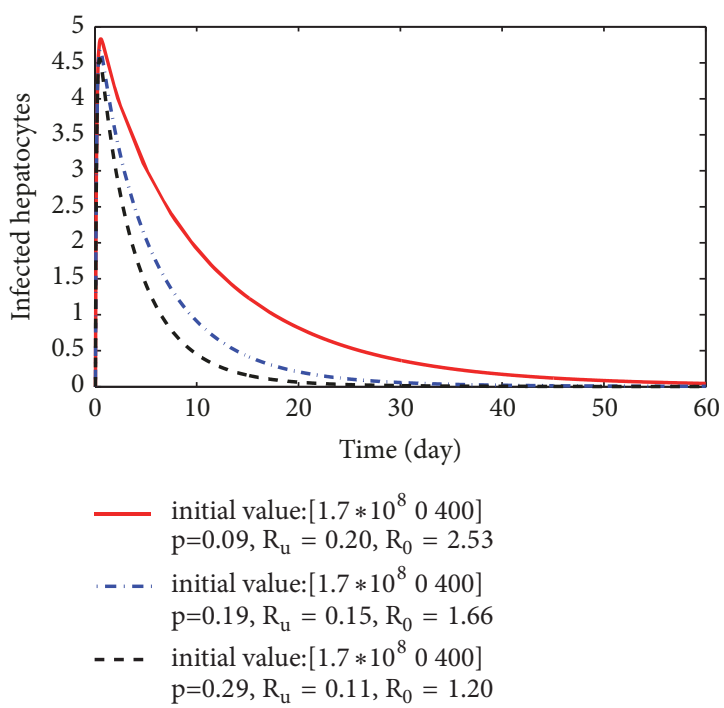

(a) $\mathrm{u}_{1}=0.2, \mathrm{u}_{2}=5, \alpha=0.95$

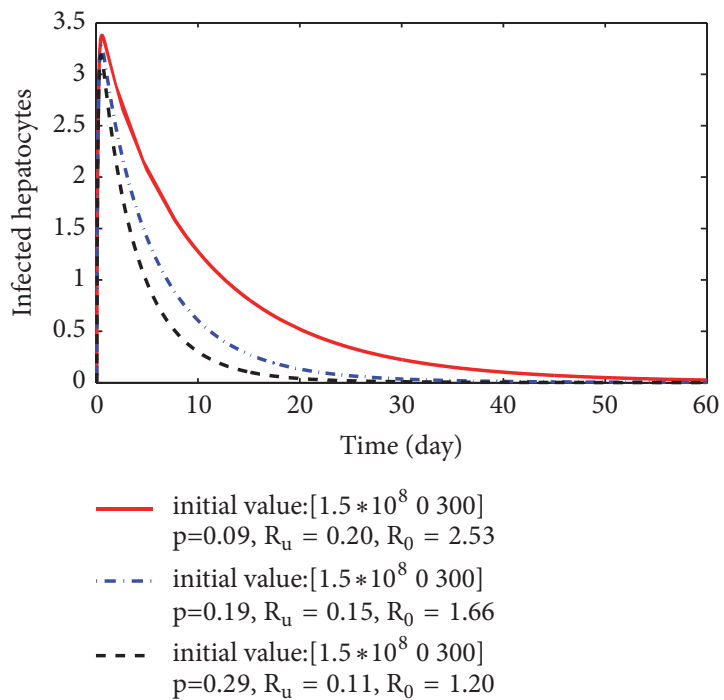

(c) $\mathrm{u}_{1}=0.2, \mathrm{u}_{2}=5, \alpha=0.95$

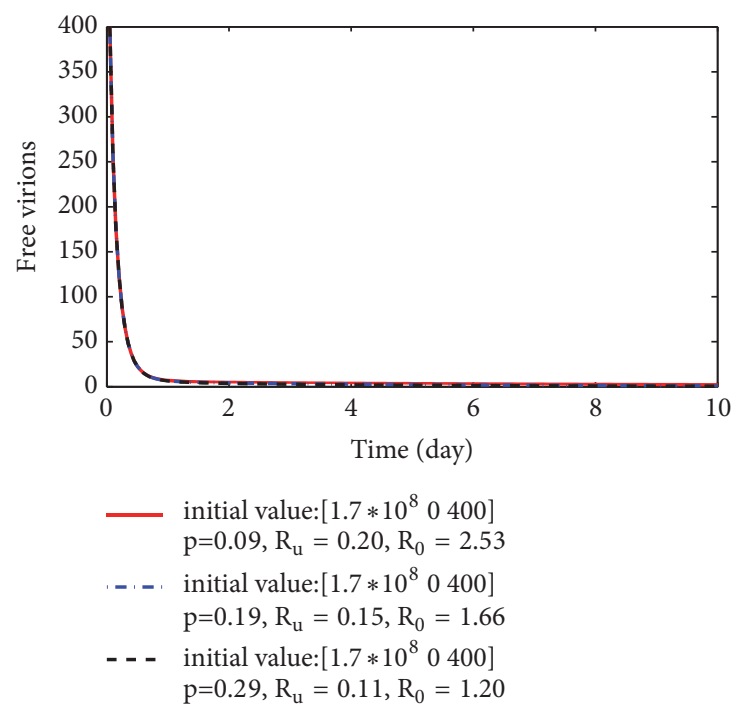

(b) $\mathrm{u}_{1}=0.2, \mathrm{u}_{2}=5, \alpha=0.95$

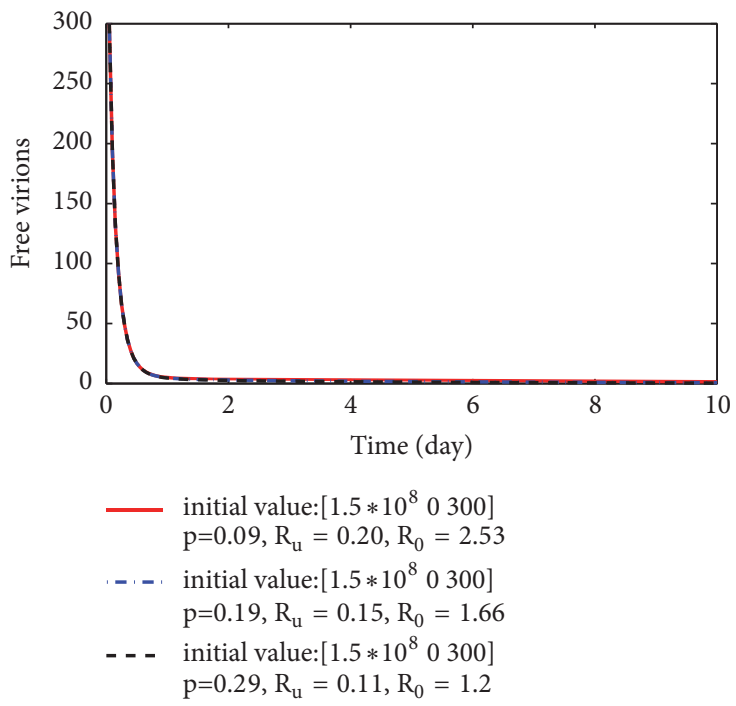

(d) $\mathrm{u}_{1}=0.2, \mathrm{u}_{2}=5, \alpha=0.95$

FIGURE 5: In this case, both IFN and LAM are used to control HBV. For different values of $p$, we can choose suitable values of $u_{1}$ and $u_{2}$ such that $R_{u}<1<R_{0}$, and these figures show that the disease-free equilibrium $E_{u}^{0}=(\Pi / \delta, 0,0)=\left(1.667 \times 10^{8}, 0,0\right)$ is asymptotically stable. The initial conditions of (a) and (b) are as follows: $\left[\begin{array}{ll}1.7 \times 10^{8} & 0400\end{array}\right]$; the initial conditions of (c) and (d) are as follows: $\left[1.5 \times 10^{8} 0300\right]$.

Remark 17. When $u_{1}=u_{2}=0, \alpha=1$ and $a=0$, the system (3) will degenerated to the model in [20].

Remark 18. From Figures 3-5, we can see that whatever the initial value are, the disease-free equilibrium $E_{u}^{0}$ is always asymptotically stable, as long as we take suitable control measures (i.e., we can take one or two control measures such that $\left.R_{u}<1\right)$.

Remark 19. By construct Lyanpunov functions, we proved the global stability of the disease-free equilibrium $E_{u}^{0}$ for system (3). However, according to Remark 16, we find the endemic equilibrium $E_{u}^{*}$ may stable or unstable; and the value of $\alpha$ is crucial for the dynamics of system (3).
Remark 20. (1) In fact, HBV can be transmitted not only from virus-to-cell, but also from cell-to-cell. In this article, only virus-to-cell is included in our model. We will consider both virus-to-cell and cell-to-cell in our future work.

(2) In this article, the fractional derivatives of the system (3) has the same order for different variables. In our future work, we will consider the dynamic behavior of models with different fractional order derivatives.

\section{Data Availability}

The data used to support the findings of this study are included within the article. 


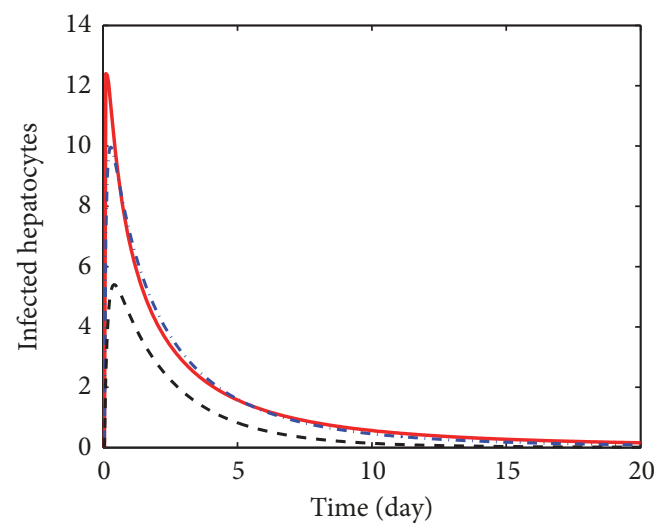

initial value: $\left[1.7 * 10^{8} 0400\right], \alpha=0.75$

_ . - initial value: $\left[1.7 * 10^{8} 0400\right], \alpha=0.85$

- - - initial value: $\left[1.7 * 10^{8} 0400\right], \alpha=0.95$

(a) $\mathrm{u}_{1}=0, \mathrm{u}_{2}=5, \mathrm{R}_{\mathrm{u}}=0.110$

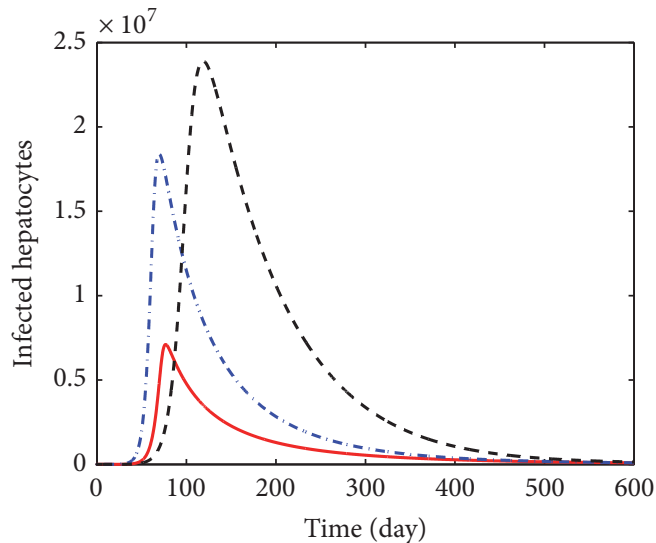

initial value: $\left[1.7 * 10^{8} 0400\right]$ alpha $=0.70$

- . - initial value: $\left[1.7 * 10^{8} 0400\right]$ alpha $=0.80$

- - - initial value: $\left[1.7 * 10^{8} 0400\right]$ alpha $=0.90$

(c) $\mathrm{u}_{1}=0.7, \mathrm{u}_{2}=0, \mathrm{R}_{\mathrm{u}}=0.768$

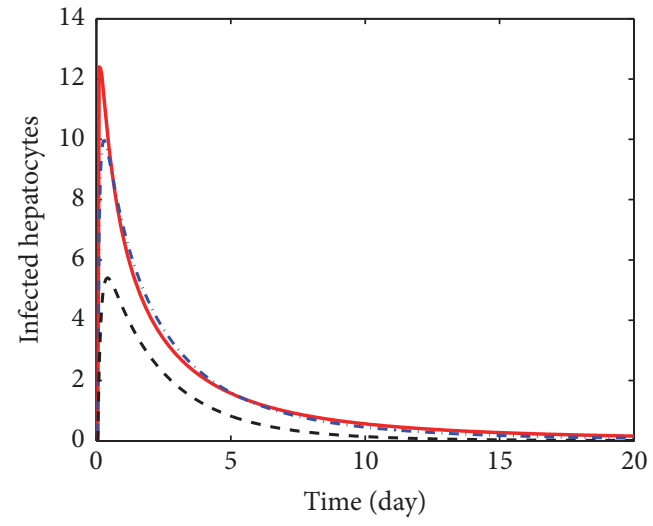

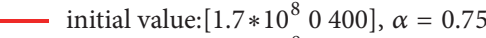

_ _ - initial value: $\left[1.7 * 10^{8} 0400\right], \alpha=0.85$

- - - initial value:[1.7*10 0400 ], $\alpha=0.95$

(e) $\mathrm{u}_{1}=0.2, \mathrm{u}_{2}=5, \mathrm{R}_{\mathrm{u}}=0.088$

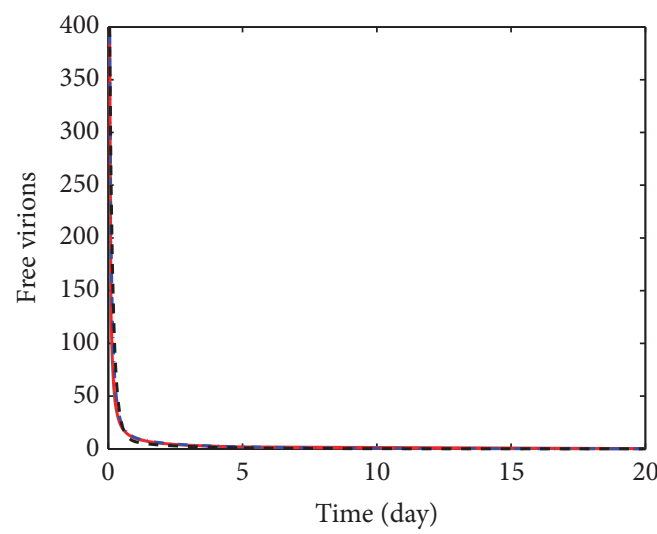

initial value: $\left[1.7 * 10^{8} 0400\right], \alpha=0.75$

_ . - initial value: $\left[1.7 * 10^{8} 0400\right], \alpha=0.85$

- - - initial value: $\left[1.7 * 10^{8} 0400\right], \alpha=0.95$

(b) $\mathrm{u}_{1}=0, \mathrm{u}_{2}=5, \mathrm{R}_{\mathrm{u}}=0.110$

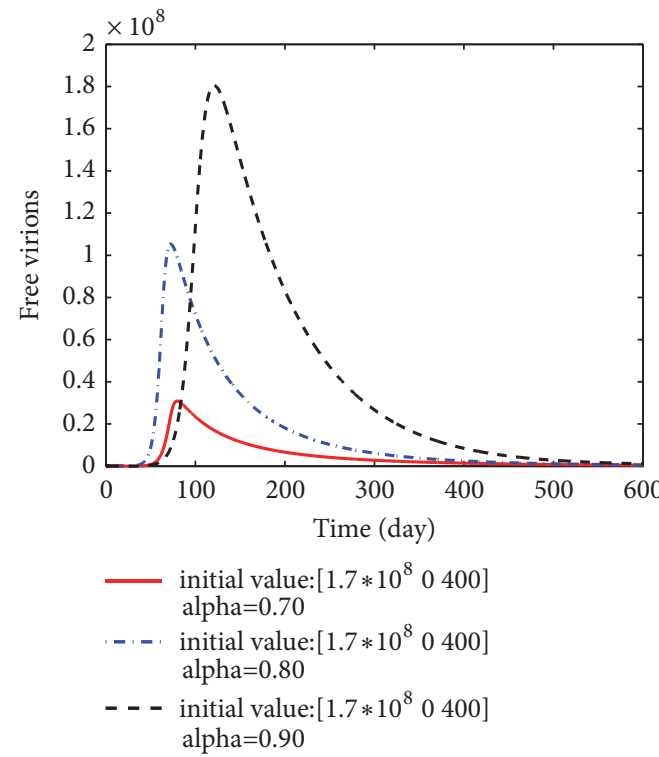

(d) $\mathrm{u}_{1}=0.7, \mathrm{u}_{2}=0, \mathrm{R}_{\mathrm{u}}=0.768$

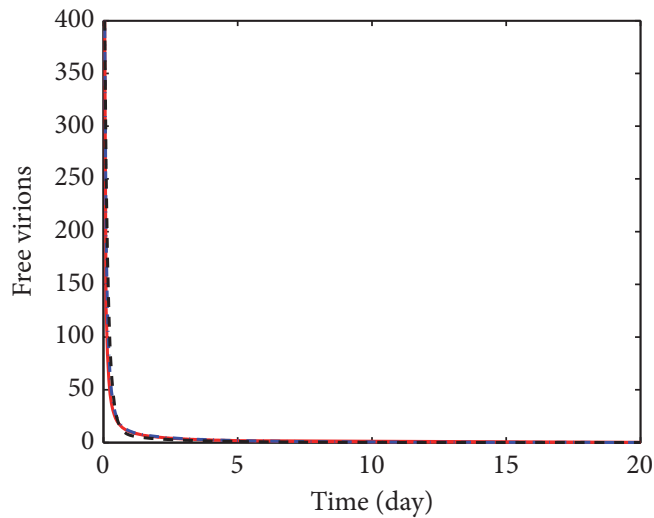

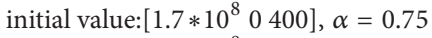

_. - . initial value: $\left[1.7 * 10^{8} 0400\right], \alpha=0.85$

- - - initial value: $\left[1.7 * 10^{8} 0400\right], \alpha=0.95$

(f) $\mathrm{u}_{1}=0.2, \mathrm{u}_{2}=5, \mathrm{R}_{\mathrm{u}}=0.088$

Figure 6: In this case, For different values of $\alpha$, when $R_{u}<1$, the disease-free equilibrium $E_{u}^{0}=(\Pi / \delta, 0,0)=\left(1.667 \times 10^{8}, 0,0\right)$ is always asymptotically stable. (a)-(b) Only LAM (without IFN) is used to treat HBV. (c)-(d) Only IFN (without LAM) is used to control HBV. (e)-(f) Both IFN and LAM are used to control HBV. 

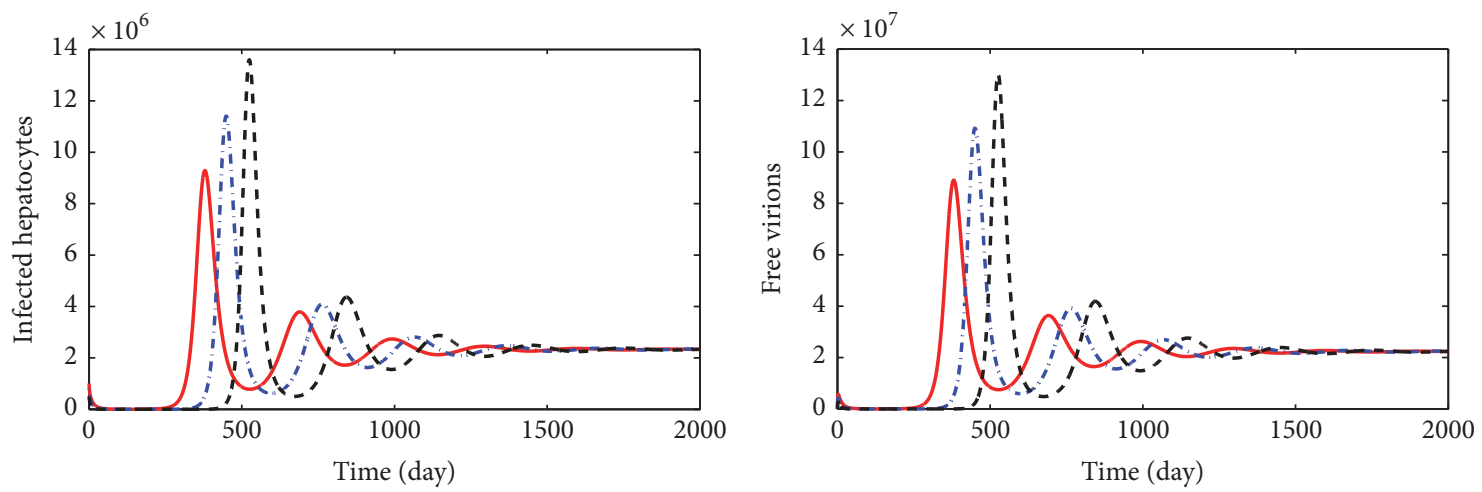

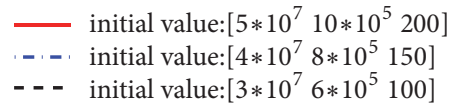

(a) $\alpha=1, \mathrm{R}_{\mathrm{u}}>1$

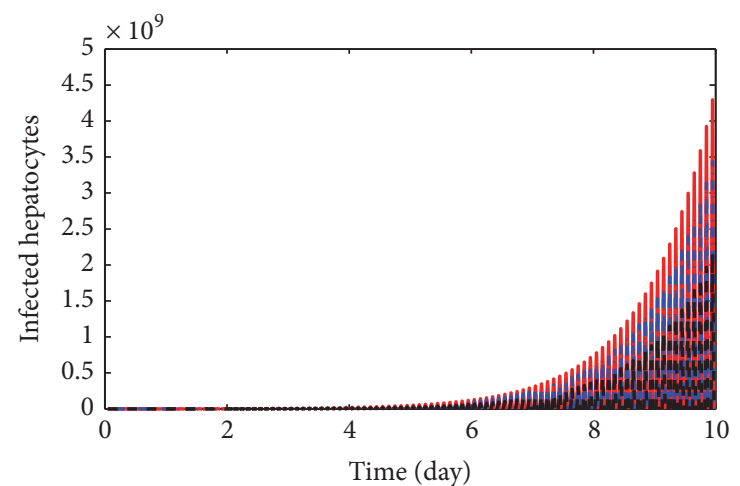

_ $\quad$ initial value: $\left[5 * 10^{7} 10 * 10^{5} 200\right]$

(c) $\alpha=0.5, \mathrm{R}_{\mathrm{u}}>1$
_ initial value: $\left[5 * 10^{7} 10 * 10^{5} 200\right]$

- . - initial value: $\left[4 * 10^{7} 8 * 10^{5} 150\right]$

- - - initial value: $\left[3 * 10^{7} 6 * 10^{5} 100\right]$

(b) $\alpha=1, \mathrm{R}_{\mathrm{u}}>1$

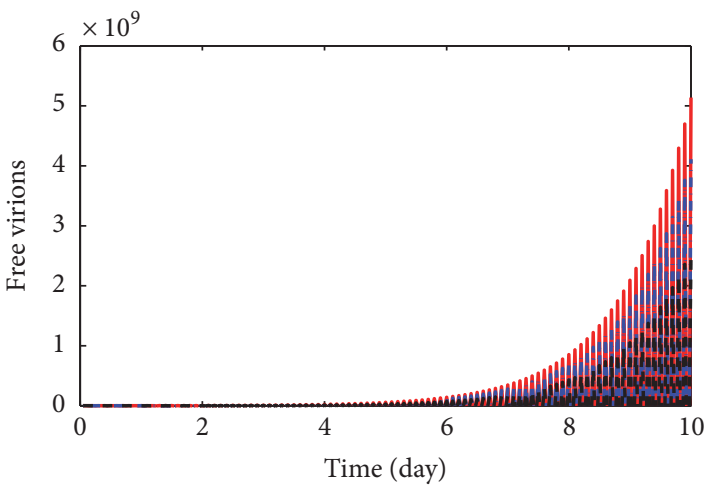

\begin{tabular}{c} 
_ initial value: $\left[5 * 10^{7} 10 * 10^{5} 200\right]$ \\
- - - $\quad$ initial value: $\left[4 * 10^{7} 8 * 10^{5} 150\right]$ \\
\hline
\end{tabular}

(d) $\alpha=0.5, \mathrm{R}_{\mathrm{u}}>1$

FIGURE 7: In this case, For different initial values: when $\alpha=1$, the endemic equilibrium $E_{u}^{*}$ is stable. However, when $\alpha=0.5$, the endemic equilibrium $E_{u}^{*}$ is unstable.

\section{Conflicts of Interest}

The authors declare that they have no conflicts of interest.

\section{Authors' Contributions}

Each of the authors, Ruiqing Shi, Ting Lu, and Cuihong Wang contributed to each part of this work equally and read and approved the final version of the manuscript.

\section{Acknowledgments}

This work is partly supported by Fund Program for the Scientific Activities of Selected Returned Overseas Professionals in Shanxi Province under Grant 2018-25.

\section{References}

[1] J. Wang, Y. Xiao, and R. A. Cheke, "Modelling the effects of contaminated environments on HFMD infections in mainland China," BioSystems, vol. 140, pp. 1-7, 2016.
[2] H. Qi, L. Liu, and X. Meng, "Dynamics of a nonautonomous stochastic SIS epidemic model with double epidemic hypothesis," Complexity, vol. 2017, Article ID 4861391, 14 pages, 2017.

[3] J. Zhang, Y. Li, Z. Jin, and H. Zhu, "Dynamics analysis of an avian influenza A (H7N9) epidemic model with vaccination and seasonality," Complexity, vol. 2019, Article ID 4161287, p. 1, 2019.

[4] A. R. Carvalho and C. M. Pinto, "Energence of drug-resistance in HIV dynamics under distict HAART regimes," Communications in Nonlinear Science and Numerical Simulation, vol. 30, no. 1-3, pp. 207-226, 2016.

[5] B. Tang, Y. Xiao, S. Tang, and J. Wu, "Modelling weekly vector control against Dengue in the Guangdong Province of China," Journal of Theoretical Biology, vol. 410, pp. 65-76, 2016.

[6] J. Lin, R. Xu, and X. Tian, "Threshold dynamics of an HIV1 virus model with both virus-to-cell and cell-to-cell transmissions, intracellular delay, and humoral immunity," Applied Mathematics and Computation, vol. 315, pp. 516-530, 2017.

[7] M. A. Nowak and C. R. M. Bangham, "Population dynamics of immune responses to persistent viruses," Science, vol. 272, no. 5258, pp. 74-79, 1996. 
[8] M. A. Nowak and R. M. May, Virus Dynamics: Mathematical Principles of Immunology and Virology, Oxford University Press, London, UK, 2000.

[9] M. A. Nowak, S. Bonhoeffer, A. M. Hill, R. Boehme, H. C. Thomas, and H. Mcdade, "Viral dynamics in hepatitis B virus infection," Proceedings of the National Acadamy of Sciences of the United States of America, vol. 93, no. 9, pp. 4398-4402, 1996.

[10] S. M. Ciupe, R. M. Ribeiro, P. W. Nelson, and A. S. Perelson, "Modeling the mechanisms of acute hepatitis B virus infection," Journal of Theoretical Biology, vol. 247, no. 1, pp. 23-35, 2007.

[11] K. Wang and W. Wang, "Propagation of HBV with spatial dependence," Mathematical Biosciences, vol. 210, no. 1, pp. 7895, 2007.

[12] A. U. Neumann, N. P. Lam, H. Dahari et al., "Hepatitis C viral dynamics in vivo and the antiviral efficacy of interferon$\alpha$ therapy," Science, vol. 282, no. 5386, pp. 103-107, 1998.

[13] A. S. Perelson, E. Herrmann, F. Micol, and S. Zeuzem, "New kinetic models for the hepatitis C virus," Hepatology, vol. 42, no. 4, pp. 749-754, 2005.

[14] WHO, Fact Sheet WHO/204 Revised October 2000: Hepatitis B, 2000, http://www.who.int/mediacentred/factsheets/fs204/en/.

[15] K. Moriyama, H. Okamoto, F. Tsuda, and M. Mayumi, "Reduced precore transcription and enhanced core-pregenome transcription of hepatitis B virus DNA after replacement of the precorecore promoter with sequences associated with e antigenseronegative persistent infections," Virology, vol. 226, no. 2, pp. 269-280, 1996.

[16] S. R. Lewin, R. M. Ribeiro, T. Walters et al., "Analysis of hepatitis B viral load decline under potent therapy: Complex decay profiles observed," Hepatology, vol. 34, no. 5, pp. 1012-1020, 2001.

[17] L. G. Guidotti, R. Rochford, J. Chung, M. Shapiro, R. Purcell, and F. V. Chisari, "Viral clearance without destruction of infected cells during acute HBV infection," Science, vol. 284, no. 5415, pp. 825-829, 1999.

[18] A. S. Perelson, "Modelling viral and immune system dynamics," Nature Reviews Immunology, vol. 2, no. 1, pp. 28-36, 2002.

[19] H. Dahari, E. Shudo, R. M. Ribeiro, and A. S. Perelson, "Modeling complex decay profiles of hepatitis B virus during antiviral therapy," Hepatology, vol. 49, no. 1, pp. 32-38, 2009.

[20] C. Vargas-De-León, "Stability analysis of a model for HBV infection with cure of infected cells and intracellular delay," Applied Mathematics and Computation, vol. 219, no. 1, pp. 389398, 2012.

[21] C. Huang, L. Cai, and J. Cao, "Linear control for synchronization of a fractional-order time-delayed chaotic financial system," Chaos, Solitons \& Fractals, vol. 113, pp. 326-332, 2018.

[22] R. Rakkiyappan, G. Velmurugan, and J. Cao, "Stability analysis of fractional-order complex-valued neural networks with time delays," Chaos, Solitons \& Fractals, vol. 78, pp. 297-316, 2015.

[23] F. A. Rihan, D. H. Abdel Rahman, and S. Lakshmanan, "A time delay model of tumour-immune system interactions: global dynamics, parameter estimation, sensitivity analysis," Applied Mathematics and Computation, vol. 232, pp. 606-623, 2014.

[24] C. Huang, J. Cao, M. Xiao, A. Alsaedi, and F. E. Alsaadi, "Controlling bifurcation in a delayed fractional predator-prey system with incommensurate orders," Applied Mathematics and Computation, vol. 293, pp. 293-310, 2017.

[25] C. M. Pinto and A. R. Carvalho, "A latency fractional order model for HIV dynamics," Journal of Computational and Applied Mathematics, vol. 312, pp. 240-256, 2017.
[26] I. Podlubny, Fractional Differential Equations: An Introduction to Fractional Derivatives, Fractional Differential Equations, to Methods of their Solution and Some of their Applications, vol. 198, Academic Press, San Diego, Calif, USA, 1999.

[27] Y. Li, D. Zhao, Y. Chen, I. Podlubny, and C. Zhang, "Finite energy Lyapunov function candidate for fractional order general nonlinear systems," Communications in Nonlinear Science and Numerical Simulation, vol. 78, 2019.

[28] F. A. Rihan, Q. M. Al-Mdallal, H. J. AlSakaji, and A. Hashish, "A fractional-order epidemic model with time-delay and nonlinear incidence rate," Chaos, Solitons \& Fractals, vol. 126, pp. 97-105, 2019.

[29] Y. Ferdi, "Some applications of fractional order calculus to design digital filters for biomedical signal processing," Journal of Mechanics in Medicine and Biology, vol. 12, no. 2, Article ID 12400088, 13 pages, 2012.

[30] L. Huo, J. Jiang, S. Gong, and B. He, "Dynamical behavior of a rumor transmission model with Holling-type II functional response in emergency event," Physica A: Statistical Mechanics and its Applications, vol. 450, pp. 228-240, 2016.

[31] V. Castellanos and R. E. Chan-López, "Existence of limit cycles in a three level trophic chain with Lotka-Volterra and Holling type II functional responses," Chaos, Solitons \& Fractals, vol. 95, pp. 157-167, 2017.

[32] X. Meng, F. Li, and S. Gao, "Global analysis and numerical simulations of a novel stochastic eco-epidemiological model with time delay," Applied Mathematics and Computation, vol. 339, pp. 701-726, 2018.

[33] Z. M. Odibat and N. T. Shawagfeh, "Generalized Taylor's formula," Applied Mathematics and Computation, vol. 186, no. 1, pp. 286-293, 2007.

[34] S. Mao, R. Xu, and Y. Li, "A fractional order sirs model with standard incidence rate," Journal of Beihua University (Natural Science), vol. 12, no. 4, pp. 79-382, 2012.

[35] P. van den Driessche and J. Watmough, "Reproduction numbers and sub-threshold endemic equilibria for compartmental models of disease transmission," Mathematical Biosciences, vol. 180, pp. 29-48, 2002.

[36] E. Ahmed, A. M. A. El-Sayed, and H. A. A. El-Saka, "On some RouthHurwitz conditions for fractional order differential equations and their applications in Lorenz, Rssler, Chua and Chen systems," Physics Letters A, vol. 358, pp. 1-4, 2006.

[37] S. M. Salman and A. M. Yousef, "On a fractional-order model for HBV infection with cure of infected cells," Journal of the Egyptian Mathematical Society, vol. 25, no. 4, pp. 445-451, 2017. 


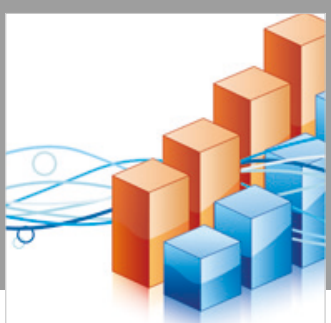

Advances in

Operations Research

\section{-n-m}
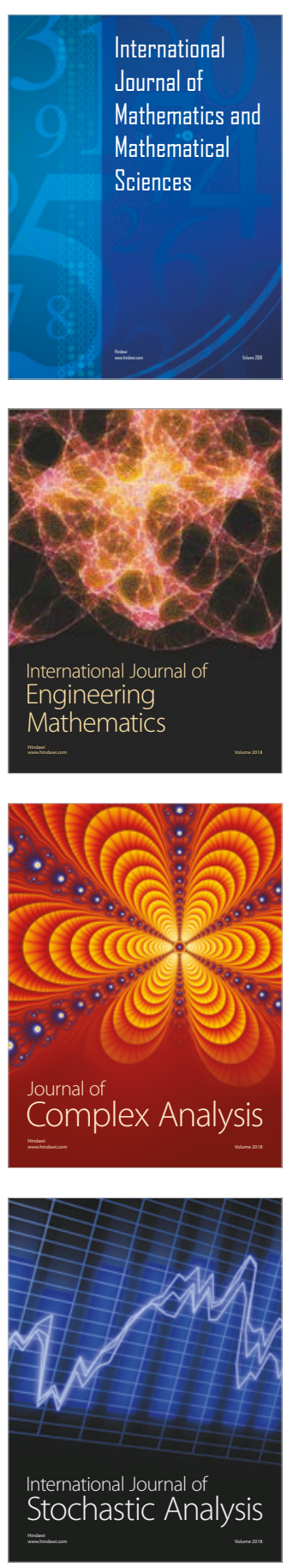
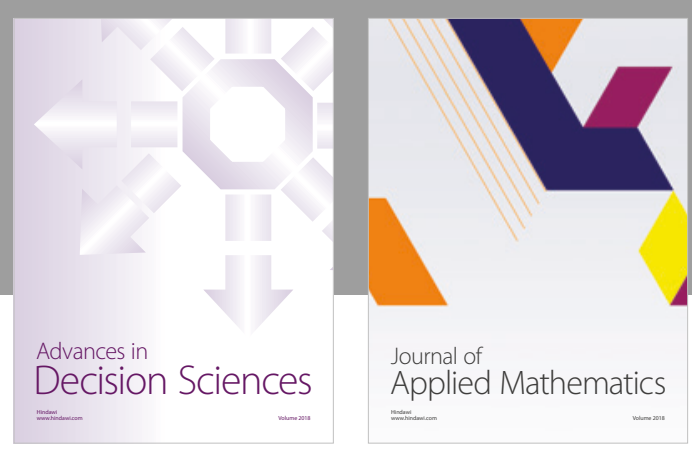

Journal of

Applied Mathematics
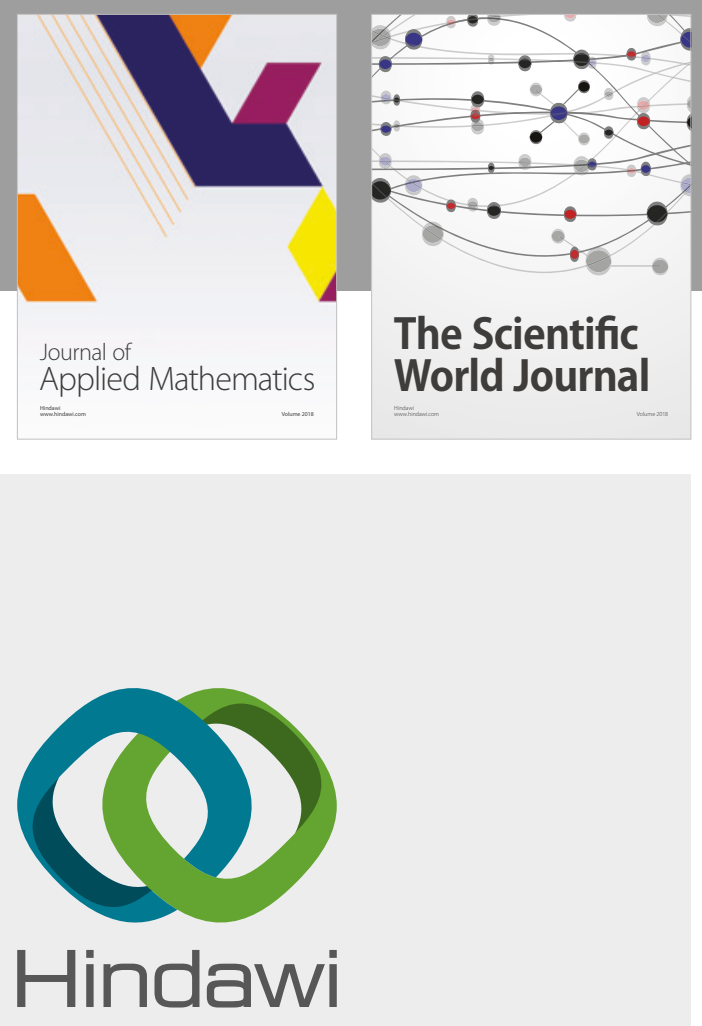

Submit your manuscripts at

www.hindawi.com

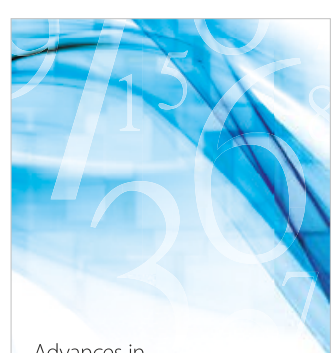

Advances in
Numerical Analysis
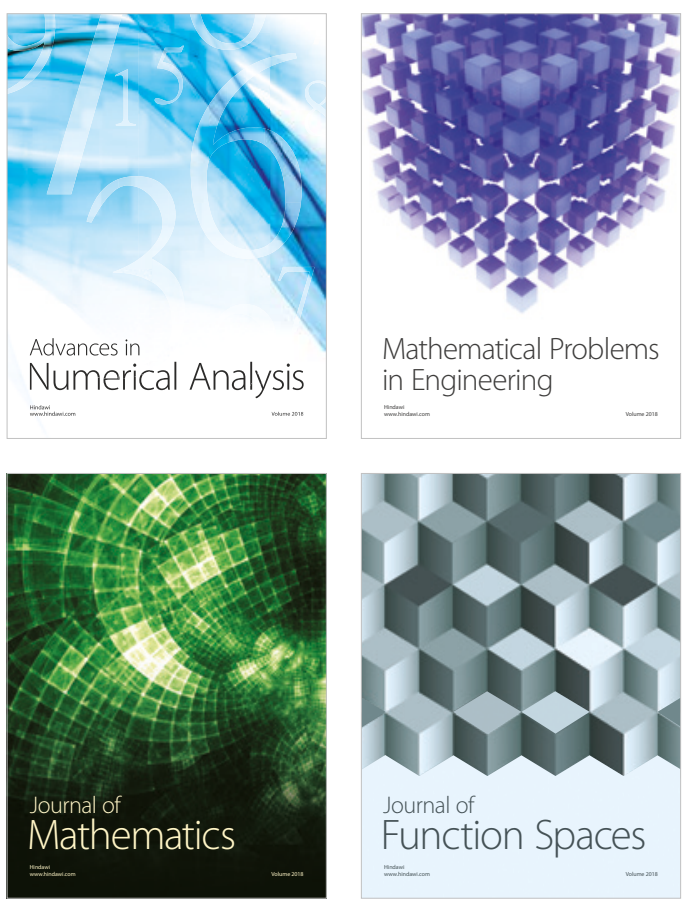

Mathematical Problems in Engineering

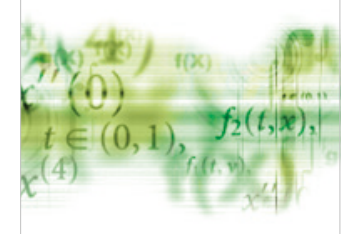

International Journal of

Differential Equations

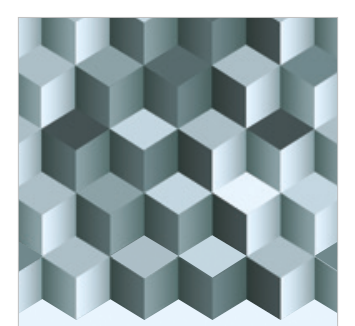

Journal of

Function Spaces
The Scientific

World Journal

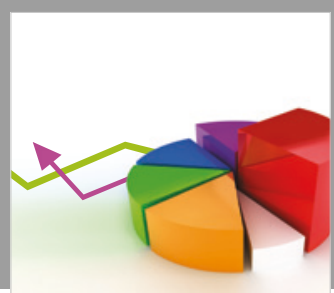

Journal of

Probability and Statistics
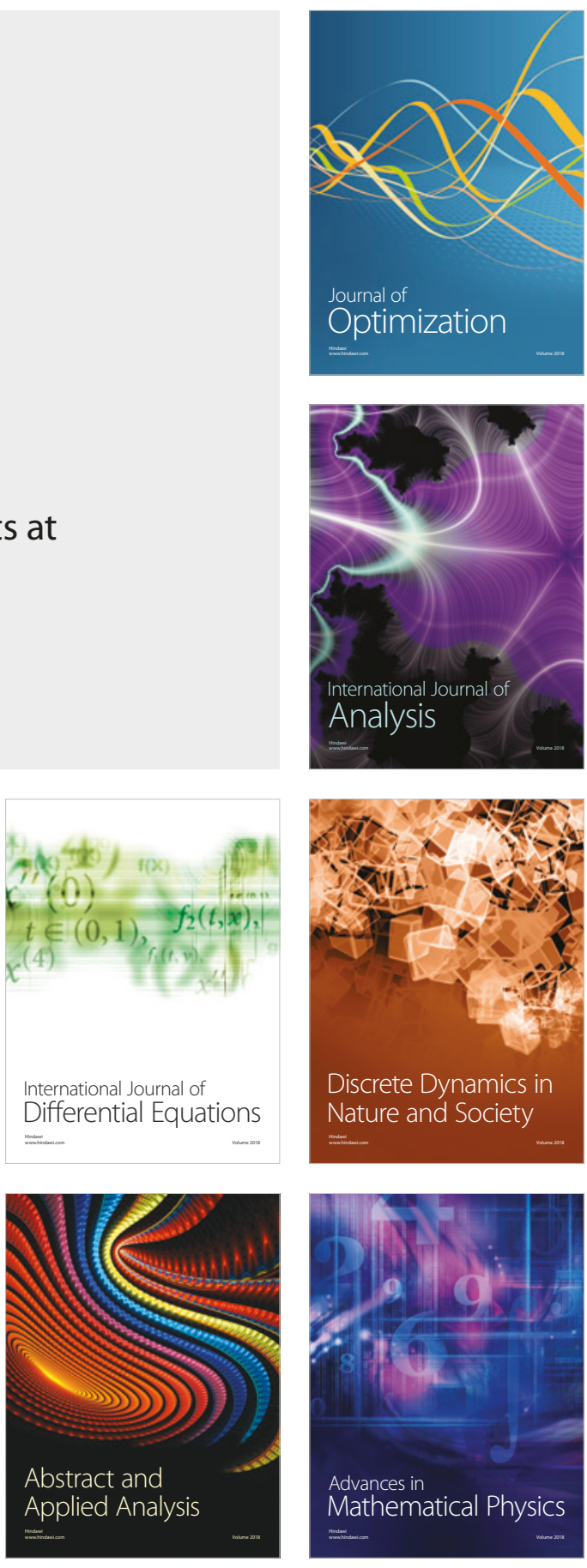